\title{
Generation of Vorticity Near the Boundary in Planar Navier-Stokes Flows *
}

\author{
G. Benfatto and M. Pulvirenti \\ Dipartimento di Matematica dell'Università di Roma La Sapienza, Piazza A. Moro, \\ I-00100 Roma, Italy
}

\begin{abstract}
We construct the solutions of the planar Navier-Stokes flow for a viscous incompressible fluid in the half-plane, by means of a boundary layer equation describing the production of vorticity on the boundary. Regularity properties are also discussed.
\end{abstract}

\section{Introduction}

The time evolution of a viscous incompressible fluid is usually described by the Navier-Stokes equations

$$
\left\{\begin{array}{l}
\frac{\partial u}{\partial t}+(u \cdot \nabla) u=v \Delta u-\nabla p+g \\
\nabla \cdot u=0
\end{array}\right.
$$

where

$$
u: D \times[0, T] \rightarrow \mathbb{R}^{d}, \quad d=2,3, \quad T>0,
$$

denotes the velocity field, $D \subset \mathbb{R}^{d}$ ( $d$ the dimension of the physical space) is an open region with smooth boundary in which the fluid is confined, $v>0$ is the viscosity coefficient, $p: D \times[0, T] \rightarrow \mathbb{R}^{1}$ is the pressure and, finally, $g: D \times[0, T] \times \mathbb{R}^{d}$ is an external force acting on the system.

Equations (1) and (2) describe the balance of momentum and conservation of mass, respectively. For simplicity the density is assumed to be one.

We are interested in the initial boundary value problem (ibvp) associated with (1) and (2). This means that we have to specify the initial value of $u$, i.e.

$$
u\left(x, 0^{+}\right)=u_{0}(x), \quad x \in D
$$

\footnotetext{
* $\quad$ Research partially supported by Ministero della Pubblica Istruzione and CNR-GNFM
} 
where $u_{0}$ is a given divergence-free vector field, and the boundary conditions. It is usually assumed that

$$
u(x, t)=0 \quad \text { for } \quad x \in \partial D,
$$

which means perfect adherence of the fluid at the boundary at rest.

The ibvp (1), (2), (4), (5) has been widely investigated and local and global unique solutions have been proved to exist for $d=3$ and $d=2$, respectively. We refer the reader to the monographs of Ladyzhenskaya and Temam [1, 2], for a comprehensive review on the subject. However, a better understanding of the structure of the solutions is desirable, even in the simpler two dimensional case. To clarify what we mean, let us first consider the case $d=2$, $\operatorname{curl} g=0$, and $D$ a domain without boundary (e.g. $D=\mathbb{R}^{2}$ or $D=T^{2}$, the two-dimensional torus). In this case the infinitely many first integrals for the Euler flow induce infinitely many a priori estimates for the Navier-Stokes flow. For example all the $L_{p}$ norm of the vorticity $\boldsymbol{\omega}=\operatorname{curl} u$ are non-increasing in time.

The situation is more involved when boundaries are present. The boundary conditions (4) are satisfied only if an extra vorticity is produced, and thus we have no more simple a priori estimates for the vorticity. Nevertheless, the decrease in time of the energy $\frac{1}{2} \int_{D} u^{2} d x$ is enough to prove existence and uniqueness of smooth solutions by means of $v$-dependent estimates.

The two situations we have mentioned are very different from a physical point of view. In particular, the vanishing viscosity limit is rather simple in the first case (in $[1,3-5]$ it is proved that the Navier-Stokes solutions converge to the corresponding Euler solutions when $v \rightarrow 0$ ), while the behavior of the flow, in the same limit and in presence of boundaries, is rather involved and a matter of debate.

Since the production of vorticity due to the boundary is a basic fact in the Navier-Stokes evolution, it is quite natural to look for rigorous estimates of the rate of such a production and to take into account this physical feature for the construction of the solution.

In this paper we do this in the case of the half-plane: the geometrical simplicity of the domain will allow us explicit calculations leading to reasonably accurate estimates.

The main idea of this paper is to describe the boundary conditions as a sort of (singular) perturbation acting on the free flow. This follows the physical idea that, to force the fluid to rest on the boundary, vorticity has to be produced by the boundary itself [6, p. 277$]$.

This approach was inspired by a numerical algorithm proposed by Chorin [7]. We will sketch the basic idea. Let $E_{t} u_{0}$ be the solution of the Euler equations with initial datum $u_{0}$. Let $\phi: u \rightarrow \tilde{u}$ be the operator which extends a vector field $u$ defined in $D$ to a vector field $\tilde{u}$ defined in a slightly larger domain $\tilde{D}$. $\tilde{u}$ has the property of the being odd across the boundary (see [8] for details). $\phi$ has essentially the effect of adding a vortex sheet concentrated on the boundary. Finally, let $H_{t}(\tilde{u})$ $=\mathbb{P}\left\{\left.\exp [t v \Delta] \tilde{u}\right|_{D}\right\}$, where $\mathbb{P}$ is the projection in $\mathbb{L}^{2}(D)$ on the divergence-free vector fields tangent to the boundary. The one-step forward operator of the Chorin algorithm is now defined as

$$
k_{\varepsilon}\left(u_{0}\right)=H_{\varepsilon} \circ \phi \circ E_{\varepsilon} u_{0} .
$$


It is easy enough to show [8] that $k_{\varepsilon}\left(u_{0}\right)$ is a consistent algorithm, that is $k_{t}\left(u_{0}\right)$ solves Eq. (1) for $t=0$ if $u_{0}=0$ on $\partial D$ and $\operatorname{div} u_{0}=0$. However, there is no convergence proof of the product formula

$$
\lim _{n \rightarrow \infty} k_{t / n}^{n}\left(u_{0}\right)=u_{t},
$$

where $u_{t}$ solves Eqs. (1)-(3).

Our method to solve the Navier-Stokes flow differs from the Chorin method, but rests on the same physical idea. In fact, we do not try to show the convergence of the product formula (7); however, our approach provides a mathematical justification of the fact that the boundary behaves as a singular source of vorticity. Hence we hope that our results could give some insight into the validity of the Chorin algorithm.

In order to realize our program, we approximate the Navier-Stokes flow by a convergent sequence of linear problems. In each linear problem the correct boundary conditions are satisfied by means of a suitable singular source (vortex sheet) of vorticity on the boundary. The main point of this paper is that the sequence of vortex sheets converges to a limit, which describes the rate of vorticity production near the boundary in the Navier-Stokes flow, Moreover, the convergence proof also implies explicit estimates of this vorticity production.

We, finally, remark that our techniques should extend, with minor modifications, to the half-space case, obviously, for short times.

The plan of the paper is the following. In Sect. 1 we deal with the Stokes flow. It is constructed following the idea explained above, perturbing the heat flow by means of a singular forcing term. The analysis can be done completely and the solutions of the Stokes problem can be written out explicitly. Among the extensive literature concerning the Stokes problem, we quote [9] for the explicit construction and regularity properties of the solutions in the half-space case (see also [10] for other considerations). Unfortunately, we cannot make use of these results for our purposes, mainly because we need different norms.

The results of Sect. 1 are generalized in Sect. 2, where a linear Navier-Stokes problem is considered. Here the transport term is given by a known drift, considered (as well as the forcing term due to the boundary conditions) as a perturbation of the heat flow. Technically speaking we use ideas of the classical theory of linear parabolic equations; however, we introduce norms that are unusual for fluid dynamical problems, but useful for our purposes.

In Sect. 3 the Navier-Stokes problem is finally constructed by means of the classical iterative method, as a limit of solutions of linear problems. Section 4 is devoted to the regularity properties of the solutions.

Most of the technical calculations and estimates are given in four appendices at the end of the paper. Very often we will interchange integrations and use integrations by parts. For the sake of brevity we do not underline the correctness of such operations whenever this is transparent from the context. Finally, we shall denote by $C$ any positive constant (not always the same) independent of the physical quantities introduced. 


\section{Stokes Equation}

The Stokes ibvp in the half-plane takes the form

$$
\left\{\begin{array}{l}
\frac{\partial}{\partial t} u(z, t)=\Delta u(z, t)-\nabla p(z, t), \\
\nabla \cdot u(z, t)=0 \\
z=(x, y) \in D=\{(x, y) \mid y>0\}, \quad u(z, t) \in \mathbb{R}^{2} \\
u\left(z, 0^{+}\right)=u_{0}(z), \quad u\left(x, 0^{+}, t\right)=0, \quad \lim _{|z| \rightarrow \infty} u(z, t)=0 .
\end{array}\right.
$$

Here $u=\left(u_{1}, u_{2}\right)$ and $p$ denote the velocity and the pressure fields, respectively, and $u_{0}$ a given divergence-free vector field. The viscosity coefficient and the density are assumed to be one for simplicity.

Introducing the vorticity $\omega=\operatorname{curl} u=\frac{\partial u_{2}}{\partial x}-\frac{\partial u_{1}}{\partial y}$ and the stream function $\Psi$

$$
u=-\nabla^{\perp} \Psi, \quad \nabla^{\perp}=\left(-\frac{\partial}{\partial y}, \frac{\partial}{\partial x}\right)
$$

( $\Psi$ exists by virtue of the incompressibility condition $\nabla \cdot u=0$ ), one easily obtains

$$
\Delta \Psi=-\omega \text {. }
$$

Rewriting the first equation in (1.1) in terms of vorticity, we have

$$
\frac{\partial}{\partial t} \boldsymbol{\omega}(z, t)=\Delta \omega(z, t),
$$

with initial value

$$
\boldsymbol{\omega}\left(z, 0^{+}\right)=\operatorname{curl} u_{0}(z) \equiv \omega_{0}(z) .
$$

To recover the boundary conditions $u\left(x, 0^{+}, t\right)=0$ and the behavior at infinity $u(z) \rightarrow 0$ as $|z| \rightarrow \infty$, one has first to specify the boundary conditions for the Laplace operator appearing in Eq. (1.3). A reasonable choice is to consider $\Delta_{D}$, the Laplacian with Dirichlet boundary conditions for which

$$
u=-\nabla^{\perp} \Delta_{D}^{-1} \omega \text {. }
$$

This ensures the correct behavior at infinity and the condition

$$
u_{2}\left(x, 0^{+}, t\right)=-\frac{\partial}{\partial x} \Delta_{D}^{-1} \omega\left(x, 0^{+}, t\right)=0
$$

The other boundary condition $u_{1}\left(x, 0^{+}, t\right)=0$ cannot follow by static considerations. Nevertheless, one has still freedom in choosing the boundary condition for the Laplace operator in Eq. (1.4). Simple calculations show that such a condition is rather complicated, because not local, hence very difficult to be handled directly. To overcome this difficulty we impose Neumann boundary conditions for the evolution problem (1.4). This choice implies that the vorticity is preserved. To take into account the vorticity produced by the boundary, according to the idea 
explained in the Introduction, we add a vortex sheet source on the boundary which will be determined by imposing the condition $u_{1}\left(x, 0^{+}, t\right)=\phi \rightarrow 0$.

To be more precise we want to make sense to the evolution problem

$$
\frac{\partial}{\partial t} \boldsymbol{\omega}(z, t)=\left(\Delta_{N} \boldsymbol{\omega}\right)(z, t)+F(z, t),
$$

where

$$
F(z, t)=f_{t}(x) \delta(y),
$$

and $f$ has to be determined by the condition $u_{1}\left(x, 0^{+}, t\right)=0$. Here $\Delta_{N}$ denotes the generator of the semigroup solving the heat equation with Neumann boundary conditions.

To perform the above program it is convenient to extend $\omega_{0}$ by parity with respect to the $y=0$ axis. Let us denote this extension again by $\omega_{0}$ and by $\omega_{0}^{+}$its restriction to the upper half-plane,

$$
\begin{aligned}
\boldsymbol{\omega}_{0}(x, y) & =\boldsymbol{\omega}_{0}^{+}(x, y) & \text { if } & y \geqq 0, \\
& =\boldsymbol{\omega}_{0}^{+}(x,-y) & \text { if } & y \leqq 0 .
\end{aligned}
$$

We shall assume $\boldsymbol{\omega}_{0}^{+}$well prepared

$$
u_{0}\left(x, 0^{+}\right)=-\nabla^{\perp} \Delta_{D}^{-1} \omega^{+}\left(x, 0^{+}\right)=0 .
$$

Equation (1.8) may be written formally

$$
\boldsymbol{\omega}(z, t)=G_{t} \boldsymbol{\omega}_{0}(z)+\int_{0}^{t}\left(G_{t-s} f_{s} \delta\right)(z),
$$

where

$$
\left(G_{t} \xi\right)(z)=\int d z^{\prime} G_{t}\left(z-z^{\prime}\right) \xi\left(z^{\prime}\right)=\int d z^{\prime} \frac{e^{-\frac{\left|z-z^{\prime}\right|^{2}}{4 t}}}{4 \pi t} \xi\left(z^{\prime}\right)
$$

denotes the free heat semigroup.

As we have noticed, $f$, the intensity of the sheet, has to be determined by the condition

$$
u_{1, t}\left(x, 0^{+}\right)=\frac{\partial}{\partial y} \Delta_{D}^{-1} \omega_{t}^{+}\left(x, 0^{+}\right)=0 .
$$

[Here and after we use indifferently the notation $\xi(\cdot, t)=\xi_{t}(\cdot)$ for all time dependent functions.]

Equation (1.14) gives rise to an equation for $f$. To deduce it we rewrite Eq. (1.14) in the following equivalent form

$$
\frac{\partial}{\partial x} \Delta^{-1} \omega_{t}(x, 0)=0 .
$$

[Here $\Delta$ and $\Delta^{-1}$ denote the Laplace operator in the whole plane and its inverse, given by the kernel $\Delta^{-1}\left(z, z^{\prime}\right)=\frac{1}{2 \pi} \ln \left|z-z^{\prime}\right|$.] 
In fact Eq. (1.14) says that the stream function $-\Delta_{D}^{-1} \omega_{t}^{+}$solves also the Neumann problem, hence cannot fail to be equal to $-\Delta_{N}^{-1} \omega_{t}^{+}=-\Delta^{-1} \omega_{t}$. The argument can obviously be reversed and the equivalence between Eqs. (1.14) and (1.15) is then established.

Combining Eqs. (1.15) and (1.12), we have:

$$
h_{t}(x)=(\mathscr{L} f)_{t}(x),
$$

where

$$
h_{t}(x)=-\frac{\partial}{\partial x} \Delta^{-1} G_{t} \omega_{0}(x, 0)
$$

and

$$
(\mathscr{L} f)_{t}(x)=\frac{\partial}{\partial x} \Delta^{-1} \int_{0}^{t} d s\left(G_{t-s} f_{s} \delta\right)(x, 0)
$$

Now, we want to show that Eq. (1.16) can be explicitly solved in suitable spaces of functions.

Let $\mathscr{B}_{1}$ be the Banach space of all continuous functions $f: \mathbb{R}^{1} \rightarrow \mathbb{R}^{1}$ of the form

$$
f(x)=\frac{1}{\sqrt{2 \pi}} \int d k e^{i k x} \hat{f}(k)
$$

equipped with the norm

$$
\|f\|=\int d k|f(k)|
$$

and

$$
\mathscr{B}_{1, \varepsilon, T}=\left\{f \in C\left((0, T], \mathscr{B}_{1}\right) \mid\|f\|_{T, \varepsilon}<\infty\right\}, \quad\|f\|_{T, \varepsilon}=\sup _{t \in(0, T]} t^{\varepsilon}\left\|f_{t}\right\| .
$$

In Appendix A we prove

Theorem 1.1. $\mathscr{L}$ is a bounded injective operator of $\mathscr{B}_{1, T, \varepsilon}$ in itself. Moreover, if $h \in \mathscr{B}_{1, T, \varepsilon}$ and satisfies the following conditions:

i) $\lim _{t \rightarrow 0} \hat{h}_{t}(k)=0$.

ii) $\hat{h}_{t}(k)$ is an absolutely continuous function of $t \in[0, T]$ for a.a. $k$.

iii) The function $f_{t}$ defined via (1.19) with

$$
\begin{gathered}
\hat{f}_{t}(k)=2 i \varepsilon(k)\left[\left(k^{2}+\frac{\partial}{\partial t}\right) \hat{h}_{t}(k)+\frac{|k|}{\sqrt{\pi}} \int_{0}^{t} \frac{d s}{\sqrt{t-s}} e^{-k^{2}(t-s)}\left(k^{2}+\frac{\partial}{\partial t}\right) \hat{h}_{s}(k)\right] \\
(\varepsilon(k)=\operatorname{sgn} k),
\end{gathered}
$$

belongs to $\mathscr{B}_{1, T, \varepsilon}$, then Eq. (1.16) has a unique solution $\mathscr{B}_{1, T, \varepsilon}$ given by Eq. (1.21).

Proof in Appendix A.

By virtue of Theorem 1.1 we could write explicitly the unique classical solution of the Stokes equation. We are not interested in doing this in all details, but one easily realizes that (1.21) can be inserted in (1.12) to give, at least, a formal solution. Regularity properties follow by the considerations in Sect. 4. 


\section{A Linear Problem}

In this section we shall deal with the following linear problem. Let $\mathscr{V}$ be the family of all time dependent continuous vector field $b: \mathbb{R} \times \mathbb{R}^{2} \rightarrow \mathbb{R}^{2}$, satisfying the following conditions:

i) $b_{t} \in C^{1}\left(\mathbb{R}_{+}^{2}\right) \cap C^{1}\left(\mathbb{R}_{-}^{2}\right) \cap C\left(\mathbb{R}^{2}\right) \quad$ for $\quad$ all $t \geqq 0$, where $\mathbb{R}_{+(-)}^{2}$ $=\left\{(x, y) \in \mathbb{R}^{2} \mid y \geqq(\leqq) 0\right\}$,

ii) $\nabla \cdot b_{t}=0$ for all $t \geqq 0$,

iii) $b_{t}=\left(b_{t}^{1}, b_{t}^{2}\right)$

$$
\begin{aligned}
& b_{t}^{1}(x, y)=b_{t}^{1}(x,-y), \\
& b_{t}^{2}(x, y)=-b_{t}^{2}(x,-y) \text { for all } t \geqq 0,
\end{aligned}
$$

iv) $b_{t}^{1}(x, 0)=0$ for all $t \geqq 0$. Notice that i) and iii) imply $b_{t}^{2}(x, 0)=0$ for all $t \geqq 0$, and that $\frac{\partial b_{t}^{1}}{\partial y}$ may be
discontinuous at $y=0$.

Let $V_{t, s}$ be a two parameter family of operators whose kernels are given by the fundamental solution associated to the parabolic linear problem with drift $b$ and diffusion coefficient 1, i.e. $V_{t, s}$ satisfies:

$$
\left\{\begin{array}{l}
\frac{\partial}{\partial t} V_{t, s}+\nabla \cdot\left(b_{t} V_{t, s}\right)=\Delta V_{t, s}, \quad t>s \geqq 0, \\
V_{s, s}=I .
\end{array}\right.
$$

Given an initial profile of vorticity $\omega_{0}^{+}$in the upper half-plane, we want to study the initial value problem

$$
\boldsymbol{\omega}_{t}(z)=\left(V_{t, 0} \boldsymbol{\omega}_{0}\right)(z)+\int_{0}^{t} d s\left(V_{t, s} F_{s}\right)(z),
$$

where $\boldsymbol{\omega}_{0}$ is given by (1.10), and where

$$
F_{s}(z)=f_{s}(x) \boldsymbol{\delta}(y), \quad z=(x, y) .
$$

$f_{s}$ has to be determined by the condition that the velocity produced by the vorticity $\boldsymbol{\omega}_{t}^{+}=\left.\boldsymbol{\omega}_{t}\right|_{y>0}$

$$
u_{t}=-\nabla^{\perp} \Delta_{D}^{-1} \omega_{t}^{+}
$$

is zero on the boundary. We denote by the same symbol $u_{t}$ its extension by reflection in the whole plane. This is given by

$$
u_{t}=-\nabla^{\perp} \Delta^{-1}\left(\omega_{t} * \varepsilon_{2}\right),
$$

where

$$
\boldsymbol{\varepsilon}_{2}(x, y)=\boldsymbol{\varepsilon}(y)=\operatorname{sgn} y .
$$

We notice that (2.6) holds since $\omega_{t}$ has the same symmetry properties of $\omega_{0}$ due to the particular choice of $b$, and $\Delta_{D}^{-1}$ can be derived, in the half-plane, by the images method. 
Proceeding as in the previous section, we couple Eq. (2.3) with the following one

$$
\frac{\partial}{\partial x}\left(\Delta^{-1} \boldsymbol{\omega}_{t}\right)(x, 0)=0
$$

that is equivalent to

$$
-h_{t}(x)+(\mathscr{L} f)_{t}(x)-(\mathscr{K} f)_{t}(x)=0
$$

where

$$
\begin{gathered}
h_{t}(x)=-\frac{\partial}{\partial x_{1}}\left(\Delta^{-1} V_{t, 0} \omega_{0}\right)(x, 0) \\
(\mathscr{K} f)_{t}(x)=-\frac{\partial}{\partial x_{1}}\left(\Delta^{-1} \int_{0}^{t} d s R_{t, s} F_{s}\right)(x, 0),
\end{gathered}
$$

and

$$
R_{t, s}=V_{t, s}-G_{t-s}
$$

[ $G$ is given by (1.13).]

As we have seen in the previous section, $\mathscr{L}^{-1}$ exists in suitable spaces and hence Eq. (2.9) can be conveniently rewritten as

$$
f_{t}-(\mathscr{T} f)_{t}=\left(\mathscr{L}^{-1} h\right)_{t},
$$

where

$$
\mathscr{T}=\mathscr{L}^{-1} \mathscr{K}
$$

and $\mathscr{L}^{-1} h$ is defined by the right-hand side of (1.21).

The above algebra has been done in view of the explicit knowledge of $\mathscr{L}^{-1}$ and the fact that $\mathscr{K}$ (which is more regular) can be estimated to give sense to $(1-\mathscr{T})^{-1}$ as a Volterra series.

Thus we want to study the initial value problem (2.3), (2.4), (2.13), (2.14). To this end we introduce the following norms:

$$
\begin{gathered}
\|f\|=\int d k|\hat{f}(k)|, \quad f: \mathbb{R}^{1} \rightarrow \mathbb{R}^{1}, \\
\|\boldsymbol{\Phi}\|=\int d k \sup _{y}|\hat{\boldsymbol{\Phi}}(k, y)|, \quad \boldsymbol{\Phi}: \mathbb{R}^{2} \rightarrow \mathbb{R}^{1},
\end{gathered}
$$

where

$$
\begin{gathered}
f(x)=\frac{1}{\sqrt{2 \pi}} \int e^{i k x} \hat{f}(k) d k, \\
\boldsymbol{\Phi}(x, y)=\frac{1}{\sqrt{2 \pi}} \int e^{i k x} \hat{\boldsymbol{\Phi}}(k, y) d k,
\end{gathered}
$$

and finally,

$$
\|b\|=\max _{i=1,2}\left\|b^{i}\right\| \quad \text { if } \quad b=\left(b^{1}, b^{2}\right): \mathbb{R}^{2} \rightarrow \mathbb{R}^{2}
$$


Accordingly to the above norms, we introduce the following Banach spaces:

$$
\begin{aligned}
\mathscr{B}_{1} & =\left\{f \in C\left(\mathbb{R}^{1}, \mathbb{R}^{1}\right) \mid\|f\|<+\infty\right\}, \\
\mathscr{B} & =\left\{\boldsymbol{\Phi} \in C\left(\mathbb{R}^{2}, \mathbb{R}^{1}\right) \mid\|\boldsymbol{\Phi}\|<+\infty\right\}, \\
\overline{\mathscr{B}} & =\{b \in \mathscr{V} \mid\|b\|<+\infty\} .
\end{aligned}
$$

For all time dependent, continuous functions $\xi:[0, T] \rightarrow \mathscr{B}_{1}, \mathscr{B}, \overline{\mathscr{B}}$ we put

$$
\|\xi\|_{T}=\sup _{t \in[0, T]}\left\|\xi_{t}\right\|,
$$

and denote by $\mathscr{B}_{1, T}, \mathscr{B}_{T}, \overline{\mathscr{B}}_{T}$ the corresponding Banach spaces [notice that $\mathscr{B}_{1, T}=\mathscr{B}_{1, T, 0}$, see Definition (1.20)]. It is also useful to introduce the following operators

$$
\begin{gathered}
\left(\mathbf{\varrho}_{t, s} \mathbf{\Phi}\right)(x, y)=\left(b_{t} \cdot \nabla V_{t, s} \boldsymbol{\Phi}\right)(x, y), \\
\left(\overline{\mathbf{\varrho}}_{t, s}^{i} \mathbf{\Phi}\right)(x, y)=\left(b_{t}^{i} V_{t, s} \mathbf{\Phi}\right)(x, y), \quad i=1,2, \\
\overline{\mathbf{\varrho}}=\left(\overline{\mathbf{\varrho}}^{1}, \overline{\mathbf{\varrho}}^{2}\right) .
\end{gathered}
$$

Lemma 2.1. Suppose $b \in \overline{\mathscr{B}}_{T}$. Let $\Phi \in \mathscr{B}$ and $T>0$. Then, for $T \geqq t>s \geqq 0$ we have:

$$
\begin{gathered}
\left\|V_{t, s} \boldsymbol{\Phi}\right\| \leqq c e^{c(t-s)\|b\|_{t}^{2}}\|\boldsymbol{\Phi}\|, \\
\left\|\overline{\boldsymbol{\varrho}}_{t, s}\right\| \mathbf{\Phi}\|\leqq c\| b\left\|_{t} e^{c(t-s)\|b\|_{t}^{2}}\right\| \mathbf{\Phi} \|, \\
\left\|\boldsymbol{\varrho}_{t, s} \boldsymbol{\Phi}\right\| \leqq \frac{c\|b\|_{t}}{\sqrt{t-s}} e^{c(t-s)\|b\|_{t}^{2}}\|\boldsymbol{\Phi}\| .
\end{gathered}
$$

Furthermore, if $\boldsymbol{\Phi}=\boldsymbol{\varphi}(x) \boldsymbol{\delta}(y)$ and $\boldsymbol{\varphi} \in \mathscr{B}_{1}$, we have

$$
\begin{gathered}
\left\|V_{t, s} \boldsymbol{\Phi}\right\| \leqq \frac{c e^{c(t-s)\|b\|_{t}^{2}}}{\sqrt{t-s}}\|\boldsymbol{\varphi}\|, \\
\left\|\overline{\boldsymbol{\varrho}}_{t, s} \boldsymbol{\Phi}\right\| \leqq \frac{c\|b\|_{t} e^{c(t-s)\|b\|_{t}^{2}}}{\sqrt{t-s}}\|\boldsymbol{\varphi}\|, \\
\left\|\boldsymbol{\varphi}_{t, s} \boldsymbol{\Phi}\right\| \leqq \frac{c\|b\|_{t} e^{c(t-s)\|b\|_{t}^{2}}}{(t-s)}\|\boldsymbol{\varphi}\| .
\end{gathered}
$$

Proof in Appendix B.

By virtue of (2.24) and (2.27), if $f \in \mathscr{B}_{1, T, 1 / 2}$ [see Definition (1.20)], $\boldsymbol{\omega}_{t}$ defined via (2.3) makes sense and $\boldsymbol{\omega} \in \mathscr{B}_{T}$ provided $b \in \overline{\mathscr{B}}_{T}$ and $\boldsymbol{\omega}_{0} \in \mathscr{B}$.

Now we show the existence of a unique $f$ satisfying (2.13) and with suitable regularity properties. As a preliminary step we estimate the operator $\mathscr{T}$.

Recalling (1.22), we have, if $f$ and $b$ are smooth enough,

where

$$
\widehat{(\widetilde{T} f)}(k)=\hat{a}_{t}(k)+\frac{|k|}{\sqrt{\pi}} \int_{0}^{t} \frac{d s}{\sqrt{t-s}} e^{-k^{2}(t-s)} \hat{a}_{s}(k),
$$

$$
\hat{a}_{t}(k)=2 i \varepsilon(k)\left[k^{2} \hat{w}_{t}(k)+\frac{\partial}{\partial t} \hat{w}_{t}(k)\right],
$$


and

$$
w_{t}(x)=\frac{1}{2 \pi} \int d x^{\prime} d y^{\prime} \frac{x^{\prime}-x}{\left(x^{\prime}-x\right)^{2}+y^{\prime 2}} \int_{0}^{t} d s R_{t, s} F_{s}\left(x^{\prime}, y^{\prime}\right) .
$$

By direct computation ( $g_{t}$ denotes the one-dimensional heat kernel)

$$
R_{t, s}^{\widehat{F}} F_{s}(k, y)=\int_{s}^{t} d \tau e^{-k^{2}(t-\tau)} \int d y^{\prime} g_{t-\tau}\left(y-y^{\prime}\right)\left(\hat{\mathbf{Q}_{\tau, s}} F_{s}\right)\left(k, y^{\prime}\right),
$$

and by the same arguments used in Appendix A

$$
\begin{aligned}
\hat{w}_{t}(k) & =\frac{1}{2 \pi} \int d y \int_{0}^{t} d s\left(R_{t, s} F_{s}\right)(k, y) \int d u e^{i k u} \frac{u}{k^{2}+y^{2}} \\
& =\frac{i}{2} \varepsilon(k) \int d y e^{-|k||y|} \int_{0}^{t} d s\left(R_{t, s}^{\hat{y}} F_{s}\right)(k, y) .
\end{aligned}
$$

Since

$$
\frac{\partial}{\partial t} R_{t, s}=-b_{t} \cdot \nabla V_{t, s}+\Delta R_{t, s}, \quad R_{s, s}=0
$$

we finally have

$$
\hat{a}_{t}(k)=\hat{a}_{t}^{1}(k)+\hat{a}_{t}^{2}(k)
$$

where

$$
\begin{gathered}
\hat{a}_{t}^{1}(k)=-\int d y e^{-|k||y|} \int_{0}^{t} d s\left(\mathbf{\varrho}_{t, s} F_{s}\right)(k, y), \\
\hat{a}_{t}^{2}(k)=-\int d y e^{-|k||y|} \int_{0}^{t} d s \int_{s}^{t} d \tau e^{-k^{2}(t-\tau)} \int d y^{\prime} \frac{\partial^{2}}{\partial y^{2}} g_{t-\tau}(y-y)\left(\varrho_{\tau, s}^{-} F_{s}\right)\left(k, y^{\prime}\right) \\
=-|k| \int d y \varepsilon(y) e^{-|k||y|} \int_{0}^{t} d s \int_{s}^{t} d \tau \frac{e^{-k^{2}(t-\tau)}}{\sqrt{t-\tau}} \int d y^{\prime} \frac{\left(y-y^{\prime}\right)}{2 \sqrt{t-\tau}} g_{t-\tau}\left(y-y^{\prime}\right)\left(\mathbf{\varrho}_{\tau, s} F_{s}\right)\left(k, y^{\prime}\right),
\end{gathered}
$$

and the last step is due to an integration by part.

Defining the operator $S$ by

$$
(S \hat{a})_{t}(k)=\frac{|k|}{\sqrt{\pi}} \int_{0}^{t} \frac{d s}{\sqrt{t-s}} e^{-k^{2}(t-s)} \hat{a}_{s}(k),
$$

we have:

Lemma 2.2. Let $b \in \overline{\mathscr{B}}_{T}$ and $f:[0, T] \times \mathbb{R}^{1} \rightarrow \mathbb{R}^{1}$ be an infinitely differentiable function of compact support. Then:

$$
\begin{gathered}
\left\|a_{t}^{i}\right\| \leqq c\|b\|_{t} e^{c t\|b\|_{t}^{2}} \int_{0}^{t} \frac{d s}{\sqrt{t-S}}\left\|f_{s}\right\|, \\
\left\|\left(S a^{i}\right)_{t}\right\| \leqq c\|b\|_{t} e^{c t\|b\|_{t}^{2}} \int_{0}^{t} \frac{d s}{\sqrt{t-S}}\left\|f_{s}\right\|, \quad i=1,2 .
\end{gathered}
$$


Proof in Appendix B.

Since by (2.30), (2.36)-(2.39)

$$
\mathscr{T}=(I+S)\left(a^{1}+a^{2}\right),
$$

Lemma 2.2 proves that $\mathscr{T}$ can be extended as a continuous operator $\mathscr{T}: \mathscr{B}_{T, \boldsymbol{\varepsilon}}$ $\rightarrow \mathscr{B}_{T, \max \left\{0, \boldsymbol{\varepsilon}-\frac{1}{2}\right\}}, \boldsymbol{\varepsilon} \in[0,1)$ if $b \in \overline{\mathscr{B}}_{T}$ and the explicit estimates (2.40) allow us to control the Volterra series defining $(1-\mathscr{T})^{-1}$.

To have estimates of $f$ in terms of $\omega_{0}$ we need first to control $\mathscr{L}^{-1} h$.

Lemma 2.3. Suppose $b \in \overline{\mathscr{B}}_{T}$, then

$$
\left\|\left(\mathscr{L}^{-1} h\right)_{t}\right\| \leqq \frac{c\left\|\boldsymbol{\omega}_{0}\right\|}{\sqrt{t}}+c\|b\|_{t} e^{c t\|b\|_{t}^{2}}\left\|\boldsymbol{\omega}_{0}\right\| .
$$

Proof in Appendix B.

We now establish the main result of this section.

Theorem 2.1. Suppose $b \in \overline{\mathscr{B}}_{T}, l \in \mathscr{B}_{1, T, \varepsilon}, \boldsymbol{\varepsilon} \in[0,1)$. Then

$$
\left\|(1-\mathscr{T})^{-1} l\right\|_{t, \varepsilon} \leqq \sum_{n \geqq 0}\left\|\mathscr{T}^{n} l\right\|_{t, \varepsilon} \leqq \gamma(t)\|l\|_{t, \varepsilon}
$$

where

$$
\gamma(t)=c \exp \left\{c t\|b\|_{t}^{2} e^{c t\|b\|_{t}^{2}}\right\} .
$$

Furthermore, if $\boldsymbol{\omega}_{0} \in \mathscr{B}$ and satisfies condition (2.8), there exists a unique solution of Eq. (2.13), $f \in \mathscr{B}_{1, T, 1 / 2}$ satisfying

$$
\left\|f_{t}\right\| \leqq \gamma(t) \frac{\left\|\boldsymbol{\omega}_{0}\right\|}{\sqrt{t}}
$$

and there exists a unique solution $\boldsymbol{\omega} \in \mathscr{B}_{T}$ of the initial value problem (2.3), (2.8).

Proof. By Lemma 2.2,

$$
\left\|(\mathscr{T} l)_{t}\right\| \leqq c\|b\|_{t} e^{c t\|b\|_{t}^{2}} \int_{0}^{t} \frac{d s}{\sqrt{t-s}}\left\|l_{s}\right\| .
$$

Hence the related Volterra series is absolutely convergent by virtue of Lemma B.1 in Appendix B, yielding Estimate (2.43). Estimate (2.45) then follows by Lemma 2.3. Finally, Estimates (2.42), (2.46) and Theorem 1.1 allow us to establish the equivalence between Eqs. (2.13) and (2.9). Thus the function $\omega_{t}$ defined in (2.3), with $f$ the solution of Eq. (2.13), satisfies the boundary condition (2.8).

In the next section we shall show how to construct the solution of the NavierStokes problem as a limit of solutions of linear problems as above, by means of the usual iterative procedure. To do this we need to prove that the velocity field generated by $\boldsymbol{\omega}_{t}^{+}$possesses the same properties of $b$.

We introduce the extensions to the whole plane of the Neumann and Dirichlet velocities generated by $\omega_{t}$ :

$$
v_{t}=-\nabla^{\perp} \Delta^{-1} \boldsymbol{\omega}_{t}, \quad u_{t}=-\nabla^{\perp} \Delta^{-1}\left(\omega_{t} * \varepsilon_{2}\right) .
$$


We have, under smoothness assumptions on $\boldsymbol{\omega}$ :

$$
\begin{gathered}
v_{t}^{1}(x, y)=\frac{1}{2 \pi} \iint d x_{1} d y_{1} \frac{y-y_{1}}{\left(x_{1}-x\right)^{2}+\left(y_{1}-y\right)^{2}} \boldsymbol{\omega}_{t}\left(x_{1}, y_{1}\right), \\
\hat{v}_{t}^{1}(k, y)=\frac{1}{2 \pi} \int d y_{1} \hat{\boldsymbol{\omega}}_{t}\left(k, y_{1}\right) \int d u \frac{y-y_{1}}{u^{2}+\left(y_{1}-y\right)^{2}} e^{i k u} \\
=\frac{1}{2} \int d y_{1} \hat{\boldsymbol{\omega}}_{t}\left(k, y_{1}\right) \boldsymbol{\varepsilon}\left(y-y_{1}\right) e^{-|k|\left|y_{1}-y\right|}, \\
v_{t}^{2}(x, y)=\frac{1}{2 \pi} \iint d x_{1} d y_{1} \frac{x_{1}-x}{\left(x_{1}-x\right)^{2}+\left(y_{1}-y\right)^{2}} \boldsymbol{\omega}_{t}\left(x_{1}, y_{1}\right), \\
\hat{v}_{t}^{2}(k, y)=\frac{1}{2 \pi} \int d y_{1} \hat{\boldsymbol{\omega}}_{t}\left(k, y_{1}\right) \int d u \frac{u}{u^{2}+\left(y_{1}-y\right)^{2}} e^{i k u} \\
=-\frac{1}{2 i} \boldsymbol{\varepsilon}(k) \int d y_{1} \hat{\boldsymbol{\omega}}_{t}\left(k, y_{1}\right) e^{-|k|\left|y_{1}-y\right|} .
\end{gathered}
$$

We remark that Eqs. (2.49) and (2.51) express the relation $\boldsymbol{\omega}_{t}=\operatorname{curl} v_{t}$ in the case in which $\left\|v_{t}\right\|<\infty$. We notice also that, by construction, $v_{t}$ and $u_{t}$ coincide in the superior half-plane (both being solutions of the Neumann and Dirichlet problem simultaneously). Furthermore, $v_{t}=-u_{t}$ for $y<0$ and hence

$$
\left\|v_{t}\right\|=\left\|u_{t}\right\|
$$

Lemma 2.4. In the hypotheses of Theorem 2.1, supposing in addition $\left\|u_{o}\right\|<\infty$, then $u \in \overline{\mathscr{B}}_{T}$ and

$$
\left\|u_{t}\right\| \leqq\left\|u_{0}\right\|+c \sqrt{t}\left\|\omega_{0}\right\| \gamma(t)
$$

Proof in Appendix B.

\section{Construction of the Solutions}

In this section we prove that the solutions of the Navier-Stokes equations can be approximated by a sequence of solutions of linear problems of the same kind as those studied in Sect. 2. In doing this we first need to investigate continuity properties of the solutions with respect to the drifts $b^{\prime} s$.

We denote by $b_{t}^{(i)}, i=1,2$ two time dependent vector fields belonging to $\overline{\mathscr{B}}_{T}$ and

$$
b_{T}=\max _{i=1,2}\left\|b^{(i)}\right\|_{T} .
$$

$T>0$ will denote an arbitrary but fixed time. We denote also by $\omega_{t}^{(i)}, V_{t, s}^{(i)}, u_{t}^{(i)}$ etc., all the objects introduced in Sect. 2, associated to the vector field $b_{t}^{(i)}$.

The technical estimates we need are summarized in the next proposition.

Proposition 3.1. Suppose $\omega_{0} \in \mathscr{B}, b^{(i)} \in \overline{\mathscr{B}}_{T}, i=1,2$. Then for $0 \leqq t \leqq T$,

$$
\begin{gathered}
\left\|f_{t}^{(1)}-f_{t}^{(2)}\right\| \leqq c \tilde{\gamma}(t)\left\|\boldsymbol{\omega}_{0}\right\|\left\|b^{(1)}-b^{(2)}\right\|_{t}, \\
\left\|\overline{\boldsymbol{\varrho}}_{t, 0}^{(1)} \boldsymbol{\omega}_{0}-\overline{\mathbf{\varrho}}_{t, 0}^{(2)} \boldsymbol{\omega}_{0}\right\| \leqq c\left\|b^{(1)}-b^{(2)}\right\|_{t} e^{c t b_{T}^{2}}\left\|\boldsymbol{\omega}_{0}\right\| .
\end{gathered}
$$


If $F_{t}(x, y)=f_{t}(x) \boldsymbol{\delta}(y), f \in \mathscr{B}_{1, T}$,

$$
\begin{gathered}
\left\|\overline{\mathbf{\varrho}}_{t, s}^{(1)} F_{s}-\overline{\mathbf{\varrho}}_{t, s}^{(2)} F_{s}\right\| \leqq c\left\|b^{(1)}-b^{(2)}\right\|_{t} \frac{e^{c t b_{T}^{2}}\left\|f_{s}\right\|}{\sqrt{t-s}}, \\
\left\|u_{t}^{(1)}-u_{t}^{(2)}\right\| \leqq \tilde{\gamma}(t)\left\|\omega_{0}\right\| \int_{0}^{t} d s\left\|b^{(1)}-b^{(2)}\right\|_{s}
\end{gathered}
$$

where

$$
\tilde{\gamma}(t)=c \exp \left\{c t b_{T}^{2} e^{c t b_{T}^{2}}\right\}
$$

Proof in Appendix C.

Let us now introduce a sequence $\left\{\boldsymbol{\omega}^{n}\right\}_{n=0}^{\infty}$ defined in the following way:

$$
\begin{aligned}
\omega_{t}^{0} & =\omega_{0}, \\
\omega_{t}^{n} & =V_{t, 0}^{n-1} \omega_{0}+\int_{0}^{t} d s V_{t, s}^{n-1} F_{s}^{n}, \quad n \geqq 1, \\
F_{s}^{n}(x, y) & =f_{s}^{n}(x) \boldsymbol{\delta}(y), \\
f_{s}^{n} & =\left(I-\mathscr{T}^{n-1}\right)^{-1} \mathscr{L}^{-1} h^{n-1},
\end{aligned}
$$

where $V_{t, s}^{n}, \mathscr{T}^{n}, h^{n}$ have been defined in Sect. 2 and the index $n$ means that they refer to the drift $b^{(n)}$, defined as

$$
\begin{gathered}
b_{t}^{(n)}=\min \left\{1, \frac{2 \sigma(T)}{\left\|u_{t}^{(n)}\right\|}\right\} u_{t}^{(n)}, \\
u_{t}^{(n)}=-\nabla^{\perp} \Delta^{-1}\left(\omega_{t}^{n} * \varepsilon_{2}\right), \quad u_{t}^{(0)} \equiv u_{0},
\end{gathered}
$$

$\boldsymbol{\sigma}(T)>\left\|\nabla^{\perp} \Delta^{-1} \omega_{0}\right\|$ to be determined later.

Theorem 3.1. Suppose $\omega_{0} \in \mathscr{B}$ and $u_{0} \in \overline{\mathscr{B}}, t \leqq T$. Then

$$
\begin{aligned}
& \lim _{\substack{n \rightarrow \infty \\
m>n}}\left\|u^{(n)}-u^{(m)}\right\|_{t}=0, \\
& \lim _{\substack{n \rightarrow \infty \\
m>n}}\left\|\omega^{n}-\omega^{m}\right\|_{t}=0 .
\end{aligned}
$$

Proof. By direct computation

$$
\left\|b_{t}^{(n)}-b_{t}^{(m)}\right\| \leqq 2\left\|u_{t}^{(n)}-u_{t}^{(m)}\right\|, \quad n, m \text { integers . }
$$

Therefore, by (3.5), for $n \geqq 2$ :

$$
\left\|u^{(n)}-u^{(n-1)}\right\|_{t} \leqq B_{T}\left\|\omega_{0}\right\| \int_{0}^{t} d s\left\|u^{(n-1)}-u^{(n-2)}\right\|_{s},
$$

where $B_{T}$ is a suitable positive constant depending only on $T$ and $\sigma(T)$. Such an inequality can be iterated to yield

$$
\left\|u^{(n)}-u^{(n-1)}\right\|_{t} \leqq \frac{\left(B_{T}\left\|\omega_{0}\right\| t\right)^{n-1}}{(n-1) !}\left\|u^{(1)}-u_{0}\right\|_{t} .
$$

Hence (3.10) is proved by using Lemma 2.4. 
The convergence of the vorticity fields follows by writing

$$
\begin{aligned}
\omega_{t}^{n}-\omega_{t}^{n-1}= & \int_{0}^{t} d s G_{t-s}\left(b_{s}^{(n-1)} \cdot \nabla V_{t, s}^{n-1}-b_{s}^{(n-2)} \cdot \nabla V_{t, s}^{n-2}\right) \omega_{0}+\int_{0}^{t} d s G_{t-s}\left(F_{s}^{n}-F_{s}^{n-1}\right) \\
& +\int_{0}^{t} d s \int_{s}^{t} d \tau G_{t-\tau}\left(b_{\tau}^{(n-1} \cdot \nabla V_{\tau-s}^{n-1} F_{s}^{(n)}-b_{\tau}^{(n-2)} \cdot \nabla V_{\tau, s}^{n-2} F_{s}^{n-1}\right) .
\end{aligned}
$$

Integrating by parts

$$
\begin{aligned}
\left\|\boldsymbol{\omega}_{t}^{n}-\boldsymbol{\omega}_{t}^{n-1}\right\| \leqq & \left.c \int_{0}^{t} \frac{d s}{\sqrt{t-s}}\left\|\left(\overline{\mathbf{\varrho}}_{s, 0}^{n-1}-\overline{\mathbf{\varrho}}_{s, 0}^{n-2}\right) \boldsymbol{\omega}_{0}\right\|+\int_{0}^{t} \frac{d s}{\sqrt{t-s}}\left\|f_{s}^{n}-f_{s}^{n-1}\right\|\right\} \\
& +\int_{0}^{t} d s \int_{s}^{t} \frac{d \tau}{\sqrt{t-\tau}}\left\{\left\|\overline{\mathbf{\varrho}}_{\tau, s}^{n-1}\left(F_{s}^{n}-F_{s}^{n-1}\right)\right\|+\left\|\left(\overline{\mathbf{\varrho}}_{\tau, s}^{n-1}-\overline{\mathbf{\varrho}}_{\tau, s}^{n-2}\right) F^{n-1}\right\|\right\},
\end{aligned}
$$

where $\overline{\mathbf{0}}^{n}$ are defined as in (2.22) with $V$ replaced by $V^{n}$. The proof is then achieved by the use of (3.14), (2.28), and Proposition 3.1.

We denote by $\omega, u, b$ the limits in $\mathscr{B}_{T}$ and $\overline{\mathscr{B}}_{T}$ of $\omega^{n}, u^{n}, b^{n}$. It is easy to realize that

$$
b_{t}=\min \left\{1, \frac{2 \sigma(T)}{\left\|u_{t}\right\|}\right\} u_{t} .
$$

Moreover, there exists $f=\lim _{n \rightarrow \infty} f_{t}^{n}$ in $\mathscr{B}_{1, T}$, and denoting by $V_{t, s}$ the family of operators defined in (2.2) relative to the drift $b$, the pair $\{\boldsymbol{\omega}, f\}$ satisfies

$$
\boldsymbol{\omega}_{t}=V_{t, 0} \boldsymbol{\omega}_{0}+\int_{0}^{t} d s V_{t, s} F_{s}, \quad F_{s}(x, y)=f_{s}(x) \delta(y)
$$

as follows by the same arguments leading to (3.16). Finally, $\left\|u_{t}\right\|<+\infty, u_{t}(x, 0)=0$, and

$$
u_{t}=-\nabla^{\perp} \Delta^{-1}\left(\omega_{t} * \varepsilon_{2}\right) \text {. }
$$

Now we prove that, for a suitable value of $\sigma(T)$, we have constructed a weak solution of the Navier-Stokes equations.

Theorem 3.2. Suppose that $\omega_{0} \in \mathscr{B}, u_{0} \in \overline{\mathscr{B}}$, and $u_{0}^{+} \in W_{2}^{2}\left(\mathbb{R}_{+}^{2}\right)$. Then the function $\omega \in \mathscr{B}_{T}$ defined in (3.18) satisfies, for any $\boldsymbol{\varphi} \in C^{\infty}\left(\mathbb{R}_{+}^{2}\right)$ of compact support vanishing on the boundary, the following equation:

$$
\frac{d}{d t}\left\langle\boldsymbol{\varphi}, \boldsymbol{\omega}_{t}\right\rangle=\left\langle\Delta \varphi, \omega_{t}\right\rangle+\left\langle\nabla \boldsymbol{\varphi}, u_{t} \omega_{t}\right\rangle
$$

The velocity field $u \in \overline{\mathscr{B}}_{T}$ defined in (3.19) satisfies, for any divergenceless $C^{\infty}$ vector field $\boldsymbol{\psi}$ of compact support vanishing on the boundary, the following equation

Here we put,

$$
\frac{d}{d t}\left\langle\boldsymbol{\psi}, u_{t}\right\rangle=\left\langle\Delta \boldsymbol{\psi}, u_{t}\right\rangle+\sum_{i, j=1}^{2}\left\langle\partial_{x_{i}} \boldsymbol{\psi}^{j}, u^{i} u^{j}\right\rangle
$$

$$
\langle a, b\rangle=\sum_{i=1}^{2}\left\langle a^{i}, b^{i}\right\rangle,
$$

$$
\langle\boldsymbol{\varphi}, \boldsymbol{\beta}\rangle=\int d k \int_{0}^{\infty} d y \overline{\hat{\boldsymbol{\varphi}}}(k, y) \hat{\boldsymbol{\beta}}(k, y) .
$$


Proof. By virtue of the estimate (2.53) there exists $T^{*}>0$ for which $b_{t}=u_{t}$, provided $\sigma(T) \geqq\left\|u_{0}\right\|$. For $0 \leqq t \leqq T^{*}$,

$$
\left\langle\boldsymbol{\varphi}, \boldsymbol{\omega}_{t}\right\rangle=\int d k \int d y \hat{\boldsymbol{\varphi}}(k, y)\left(V_{t, 0} \boldsymbol{\omega}_{0}\right)(k, y)+\int_{0}^{t} d s \int d k d y \hat{\boldsymbol{\varphi}}(k, y)\left(V_{t, s} F_{s}\right)(k, y),
$$

and (3.20) follows by integrating by parts, once we prove

In fact,

$$
\lim _{t \rightarrow s}\left\langle\boldsymbol{\varphi}, V_{t, s} F_{s}\right\rangle=0 \text {. }
$$

$$
\left\langle\boldsymbol{\varphi}, V_{t, s} F_{s}\right\rangle=\left\langle\boldsymbol{\varphi}, G_{t-s} F_{s}\right\rangle+\int_{s}^{t} d \tau\left\langle\varphi, G_{t-\tau} u_{\tau} \cdot \nabla V_{\tau, s} F_{s}\right\rangle .
$$

The first term in the right-hand side of (3.26) goes to zero as $t \rightarrow s$ since

$$
\int \hat{\boldsymbol{\varphi}}(k, y) g_{t-s}(y) \underset{t \rightarrow s}{\longrightarrow} \hat{\boldsymbol{\varphi}}(k, 0)=0 .
$$

On the other hand,

$$
\begin{aligned}
\left|\left\langle\boldsymbol{\varphi}, G_{t-\tau} u_{\tau} \cdot \nabla V_{\tau, s} F_{s}\right\rangle\right| & \leqq\left|\left\langle\nabla \boldsymbol{\varphi}, G_{t-\tau} u_{\tau} V_{\tau, s} F_{s}\right\rangle\right| \\
& \leqq d y \sup _{k}|\nabla \hat{\boldsymbol{\varphi}}|(k, y)\left\|\overline{\mathbf{\varrho}}_{\tau, s} F_{s}\right\| .
\end{aligned}
$$

Using Estimate (2.28) in (3.28) we finally obtain (3.25). Equation (3.21) is now a straightforward consequence of (3.20). Denoting $\boldsymbol{\psi}=-\nabla^{\perp} \boldsymbol{\varphi}$, by integrating by parts we get

$$
\begin{gathered}
\left\langle\boldsymbol{\varphi}, \operatorname{curl} u_{t}\right\rangle=-\left\langle\nabla^{\perp} \boldsymbol{\varphi}, u_{t}\right\rangle=\left\langle\boldsymbol{\psi}, u_{t}\right\rangle, \\
\left\langle\Delta \boldsymbol{\varphi}, \operatorname{curl} u_{t}\right\rangle=-\left\langle\Delta \nabla^{\perp} \boldsymbol{\varphi}, u_{t}\right\rangle=-\left\langle\Delta \boldsymbol{\psi}, u_{t}\right\rangle, \\
\left\langle\nabla \boldsymbol{\varphi}, u_{t} \operatorname{curl} u_{t}\right\rangle=-\left\langle\nabla \boldsymbol{\varphi},(u \cdot \nabla) u^{\perp}\right\rangle+\frac{1}{2}\left\langle\nabla \boldsymbol{\varphi}, \nabla^{\perp} u^{2}\right\rangle,
\end{gathered}
$$

where the last step is due to the identity:

$$
\frac{1}{2} \nabla^{\perp} u^{2}=u \operatorname{curl} u+(u \cdot \nabla) u^{\perp} .
$$

The last term in (3.31) obviously equals zero, while the first one may be rewritten as $\left\langle\nabla^{\perp} \boldsymbol{\varphi},(u \cdot \nabla) u\right\rangle$, yielding (3.21). Thus we have proved that, at least for $t \leqq T^{*}$, our solution coincides with a weak solution of Navier-Stokes equations. Furthermore, this solution is the only one for which $\boldsymbol{\omega}_{t} \in \mathscr{B}_{T}$, as easily follows by our previous considerations. To extend this solution for all times we need an a priori estimate (basically the energy bound).

It is well known that, under our hypotheses on $u_{0}$, the following holds (in two dimensions!)

$$
\begin{gathered}
u^{+}, \partial_{t} u^{+}, \partial_{x_{i}} u^{+} \in L_{\infty}\left([0, T], L_{2}\left(\mathbb{R}_{+}^{2}\right)^{2}\right), \\
u^{+} \in L_{\infty}\left([0, T],\left(L_{4}\left(\mathbb{R}_{+}^{2}\right)\right)^{2}\right), \quad u^{+}=\left.u\right|_{y>0} .
\end{gathered}
$$

(See [1] and [2].)

We want to prove, by the use of (3.33), the existence of a continuous function $\boldsymbol{\alpha}(t)$ for which

$$
\left\|u_{t}\right\| \leqq \alpha(t)
$$


and this would achieve the proof of the theorem, by choosing $\sigma(T)>\frac{\alpha(T)}{2}$. Actually, with such a choice of $\sigma(T)$, convergence of the same kind of (3.14) will hold for the approximating linear problems solutions to the Navier-Stokes solutions.

To prove (3.34), we observe that $u_{t}^{+}$satisfies, as a distribution on $\mathbb{R}_{+}^{2}$ for almost all $t>0$, the equation

$$
(1-\Delta) u_{t}^{+}=-\nabla p_{t}+h_{1}+h_{2},
$$

where $h_{1}=u_{t}-\partial_{t} u \in L_{2}\left(\mathbb{R}_{+}^{2}\right)^{2}, h_{2}=(u \cdot \nabla) u \in L_{4 / 3}\left(\mathbb{R}_{+}^{2}\right)^{2}, p$ is the pressure. This

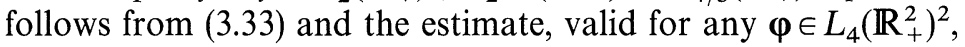

$$
\left|\left(\boldsymbol{\varphi}, h_{2}\right)\right|=\left|\int d z \boldsymbol{\varphi}(z)\left[\left(u_{t} \cdot \nabla\right) u_{t}\right](z)\right| \leqq 4\|\boldsymbol{\varphi}\|_{4}\left\|u_{t}\right\|_{4}\left\|\nabla u_{t}\right\|_{2} .
$$

The bound (3.34) then follows by the use of the following lemma which will be proved in Appendix C.

Lemma 3.1. If the field $u^{+}$satisfies the following equations

$$
\left\{\begin{array}{l}
(1-\Delta) u^{+}=\nabla p+h^{+}, \\
u^{+}(x, 0)=0, \\
\nabla \cdot u^{+}=0, \quad y>0,
\end{array}\right.
$$

and $h^{+} \in L_{p}\left(\mathbb{R}_{+}^{2}\right)^{2}, 1<p \leqq 2$, then $u^{+}$is the restriction to $\mathbb{R}_{+}^{2}$ of a function $u \in \overline{\mathscr{B}}$.

\section{Regularity Properties}

In this section we want to investigate further regularity properties of the solution we have constructed. It is not hard to prove that, under suitable assumptions on the initial datum $\boldsymbol{\omega}_{0}, \boldsymbol{\omega}_{t}$, and $u_{t}$ are classical solutions of the Navier-Stokes equations, since, out of the boundary, all the derivatives we need make sense. Our main effort here, is to prove uniform estimates up to the boundary, on first and second derivatives of vorticity and velocity. Such estimates are, in a sense, optimal, having the same structure of the corresponding estimates for the Stokes flow.

We need to introduce the following Banach spaces:

$$
\begin{aligned}
\mathscr{B}_{1}^{(n)}= & \left\{f \in C^{n}\left(\mathbb{R}^{1}, \mathbb{R}^{1}\right) \mid\|f\|^{(n)}=\max _{0 \leqq i \leqq n}\left\|\partial_{x}^{n} f\right\|<+\infty\right\}, \\
\mathscr{B}^{(n, m)}= & \left\{\boldsymbol { \Phi } \in C \left(\mathbb{R}^{2}, \mathbb{R}^{1)} \mid \partial_{x}^{n} \partial_{y}^{m} \boldsymbol{\Phi} \in C\left(\mathbb{R}_{ \pm}^{2}, \mathbb{R}^{1}\right),\right.\right. \\
& \left.\|\boldsymbol{\Phi}\|^{(n, m)}=\max _{\substack{0 \leqq i \leqq n \\
0 \leqq j \leqq m}}\left\|\partial_{x}^{n} \partial_{y}^{m} \boldsymbol{\Phi}\right\|<+\infty \text { and } \boldsymbol{\Phi}(x, y)=\boldsymbol{\Phi}(x,-y)\right\}, \\
\overline{\mathscr{B}}^{(n, m)}= & \left\{b \in \mathscr{V} \mid \partial_{x}^{n} \partial_{y}^{m} b \in C\left(\mathbb{R}_{ \pm}^{2}, \mathbb{R}^{2}\right),\|\mathrm{b}\|^{(n, m)}=\max _{\substack{0 \leqq i \leqq n \\
0 \leqq j \leqq m}}\left\|\partial_{x}^{n} \partial_{y}^{m} b\right\|<+\infty\right\} .
\end{aligned}
$$

Notice that the functions belonging to $\mathscr{B}^{(n, m)}$ and $\overline{\mathscr{B}}^{(n, m)}$ may have a discontinuity of the $y$-derivative in $y=0$. 
We also define $\mathscr{B}_{1, T}^{(n)}=C\left([0, T], \mathscr{B}_{1}^{(n)}\right)$, and analogously $\mathscr{B}_{T}^{(n, m)}$ and $\overline{\mathscr{B}}_{T}^{(n, m)}$.

The main point in the following discussion will be a simple lemma about the regularity properties of the most singular term appearing in the expression of $\omega_{t}$, Eq. (3.18), that is the function

$$
\mathscr{H}_{t}=\int_{0}^{t} d s G_{t-s} F_{s} .
$$

Lemma 4.1. If $f \in \mathscr{B}_{1, T}^{(1)}$ and for some $k>0, \boldsymbol{\alpha}>\frac{1}{2}, 0 \leqq \boldsymbol{\beta}<1,0 \leqq s \leqq t \leqq T$,

$$
\left\|f_{t}-f_{s}\right\|^{(0)} \leqq \frac{k}{s^{\beta}}(t-s)^{\alpha},
$$

then $\mathscr{H}_{t} \in \mathscr{B}^{(1,1)} \cap \mathscr{B}^{(0,2)}$ for $t>0$ and

$$
\begin{gathered}
\left\|\mathscr{H}_{t}\right\|^{(1,1)} \leqq \frac{1}{2}\left\|f_{t}\right\|^{(1)}+c \int_{0}^{t} \frac{d s}{(t-s)^{3 / 2}}\left\|f_{t}-f_{s}\right\|^{(0)}, \\
\left\|\partial_{y y}^{2} \mathscr{H}_{t}\right\|^{(0,0)} \leqq c\left(\frac{1}{\sqrt{t}}+1\right)\left\|f_{t}\right\|^{(1)}+c \int_{0}^{t} \frac{d s}{(t-s)^{3 / 2}}\left\|f_{t}-f_{s}\right\|^{(0)} .
\end{gathered}
$$

Proof in Appendix D.

To use Lemma 4.1 we have to find conditions on $\boldsymbol{\omega}_{0}$ implying Estimate (4.3) for the function $f$ which appears in Eq. (3.18). This function satisfies Eq. (2.13), where the operator $\mathscr{T}$ and the function $h$ are expressed in terms of $u$, a solution of the Navier-Stokes equations. Then we have to study the $t$-continuity of $(\mathscr{T} f)_{t}$ and $\left(\mathscr{L}^{-1} h\right)_{t}$.

In Appendix D we prove the following lemmas.

Lemma 4.1. If $\boldsymbol{\omega}_{0} \in \mathscr{B}^{(2,1)}$ and $b \in \overline{\mathscr{B}}_{T}^{(2,1)}, 0 \leqq s \leqq t \leqq T$,

$$
\left\|\left(\mathscr{L}^{-1} h\right)_{t}-\left(\mathscr{L}^{-1} h\right)_{s}\right\|^{(0)} \leqq c(T)\left[\frac{t-s}{\sqrt{s}}+\left\|b_{t}-b_{s}\right\|^{(1,0)}\right],
$$

where $c(T)$ depends only on $T, \boldsymbol{\omega}_{0}, b$.

Lemma 4.3. If $\boldsymbol{\omega}_{0}=\operatorname{curl} u_{0} \in \mathscr{B}^{(2,0)} \cap \mathscr{B}^{(1,1)}, u_{0} \in \overline{\mathscr{B}}$ and $u_{0}^{+} \in W_{2}^{2}\left(\mathbb{R}_{+}^{2}\right)$, the solution $u$ of the Navier-Stokes equations, satisfies for any $\varepsilon \in\left(0, \frac{1}{2}\right]$ the estimate

$$
\left\|u_{t}-u_{s}\right\|^{(1,0)} \leqq c(T) \frac{(t-s)^{1-\varepsilon}}{\varepsilon}, \quad 0 \leqq s \leqq t \leqq T .
$$

Lemma 4.4. If $b \in \overline{\mathscr{B}}_{T}^{(2,0)}$, the operator $\mathscr{T}$, defined in (2.30), satisfies the following estimate, for any $\varepsilon \in\left(0, \frac{1}{2}\right], 0 \leqq s \leqq t \leqq T$,

$$
\left\|(\mathscr{T} f)_{t}-(\mathscr{T} f)_{s}\right\|^{(0)} \leqq c(T)\left[\frac{(t-s)^{1-\varepsilon}}{\varepsilon}+\left\|b_{t}-b_{s}\right\|^{(1,0)}\right]\left\|f_{t}\right\|^{(2)} .
$$

Lemma 4.5. If $\boldsymbol{\omega}_{0} \in \mathscr{B}^{(2,1)}$ and $b \in \overline{\mathscr{B}}_{T}^{(2,1)}$, the solution $f$ of $E q$. (2.13) satisfies, for any $\varepsilon \in\left(0, \frac{1}{2}\right]$, the following estimate

$$
\left\|f_{t}-f_{s}\right\|^{(0)} \leqq c(T)\left[\frac{(t-s)^{1-\varepsilon}}{\varepsilon \sqrt{s}}+\left\|b_{t}-b_{s}\right\|^{(1,0)}\right] .
$$


Moreover, if $\omega_{0} \in \mathscr{B}^{(n, 1)}$ and $b \in \overline{\mathscr{B}}_{T}^{(n, 0)}, f \in \mathscr{B}_{1, T}^{(n)}, \forall n \geqq 0$.

We are in position to establish the main result of this section.

Theorem 4.1. Suppose $\omega_{0}=\operatorname{curl} u_{0} \in \mathscr{B}^{(2,1)} \cap \mathscr{B}^{(0,2)}, u_{0} \in \overline{\mathscr{B}}, u_{0}^{+} \in W_{2}^{2}\left(\mathbb{R}_{+}^{2}\right)$, and denote by $u$ and $\boldsymbol{\omega}$ the solutions of the Navier-Stokes equations with $u_{0}$ as initial datum. Then there exists $c(T)>0$ depending only on $\omega_{0}$ and $T$ such that

$$
\begin{gathered}
\|u\|_{T}^{(2,1)}+\|u\|_{T}^{(0,2)}+\|u\|_{T}^{(3,0)}+\left\|\partial_{t} u\right\|_{T}^{(0,0)} \leqq c(T), \\
\|\boldsymbol{\omega}\|_{T}^{(2,0)}+\|\boldsymbol{\omega}\|_{T}^{(0,1)} \leqq c(T), \\
\|\boldsymbol{\omega}\|_{T}^{(0,2)}+\left\|\partial_{t} \boldsymbol{\omega}_{t}\right\|^{(0,0)} \leqq c(T) / \sqrt{t}
\end{gathered}
$$

Proof. Putting $\boldsymbol{\delta}=\frac{\partial^{n+m}}{\partial x^{n} \partial y^{m}}, n, m \geqq 0$, by (3.18) we obtain

$$
\delta \boldsymbol{\omega}_{t}=\delta G_{t} \boldsymbol{\omega}_{0}+\int_{0}^{t} d s \delta G_{t-s} F_{s}+\int_{0}^{t} d s \delta G_{t-s}\left(u_{s} \cdot \nabla\right) \omega_{s}
$$

and hence for $\boldsymbol{\delta}=\frac{\partial}{\partial x}$,

$$
\begin{aligned}
\left\|\frac{\partial \boldsymbol{\omega}_{t}}{\partial x}\right\| \leqq & \left\|\boldsymbol{\omega}_{0}\right\|^{(1,0)}+c \int_{0}^{t} \frac{d s}{\sqrt{t-s}}\left\|f_{s}\right\|^{(1)}+c \int_{0}^{t} \frac{d s}{\sqrt{t-s}}\left\|\boldsymbol{\omega}_{s}\right\|\left\|u_{s}\right\|^{(1,0)} \\
& +c\|u\|_{T} \int_{0}^{t} \frac{d s}{\sqrt{t-s}}\left\|\frac{\partial \boldsymbol{\omega}_{s}}{\partial x}\right\| .
\end{aligned}
$$

Therefore, $\omega \in \mathscr{B}_{T}^{(1,0)}$ if $u \in \overline{\mathscr{B}}_{T}^{(1,0)}$ and $\omega_{0} \in \mathscr{B}^{(1,0)}$, since, by Lemma 4.5, $f \in \mathscr{B}_{1, T}^{(1)}$. By putting $\boldsymbol{\delta}=\frac{\partial^{2}}{\partial x^{2}}$, repeating the argument, we can conclude that $\boldsymbol{\omega} \in \mathscr{B}_{T}^{(2,0)}$ if $u \in \overline{\mathscr{B}}_{T}^{(2,0)}$ and $\omega_{0} \in \mathscr{B}^{(2,0)}$.

Let us denote the above argument by $\mathrm{A}$.

Furthermore, we have already proved in Appendix B [Estimates (B.42), (B.45)] that $u \in \mathscr{B}^{(1,0)} \cap \mathscr{B}^{(0,1)}$ if $\omega \in \mathscr{B}_{T}$. We denote this argument by $\mathrm{B}$. Finally, by $(2.49)$ and (2.51) one easily proves that $u \in \overline{\mathscr{B}}_{T}^{(n, 0)}$, provided $\omega \in \overline{\mathscr{B}}_{T}^{(n-1,0)}, n \geqq 1$. We refer to this as argument $\mathrm{C}$. Then, the following chain of implications is true:

$$
\begin{aligned}
& \omega_{0} \in \mathscr{B}^{(2,0)} \Rightarrow u \in \overline{\mathscr{B}}_{T}^{(1,0)}, \quad \omega_{0} \in \mathscr{B}^{(2,0)} \Rightarrow \omega \in \mathscr{B}_{T}^{(1,0)}, \\
& \omega_{0} \in \mathscr{B}^{(2,0)} \underset{\mathrm{C}}{\stackrel{\mathrm{B}}{\Rightarrow}} u \in \overline{\mathscr{B}}_{T}^{(2,0)}, \quad \omega_{0} \in \mathscr{B}^{(2,0)} \underset{\mathrm{A}}{\stackrel{\mathrm{A}}{\Rightarrow}} \boldsymbol{\omega}\left(\mathscr{B}_{T}^{(2,0)} \underset{\mathrm{C}}{\Rightarrow} u \in \overline{\mathscr{B}}_{T}^{(3,0)}\right. \text {. }
\end{aligned}
$$

To estimate the $y$-derivatives we make again use of (4.13) with $\boldsymbol{\delta}=\frac{\partial}{\partial y}$ to obtain:

$$
\frac{\partial}{\partial y} \boldsymbol{\omega}_{t}=\frac{\partial}{\partial y} G_{t} \boldsymbol{\omega}_{0}+\frac{\partial}{\partial y} \mathscr{H}_{t}+\int_{0}^{t} d s \nabla G_{t-s} \cdot \frac{\partial u_{s}}{\partial y} \boldsymbol{\omega}_{s}+\int_{0}^{t} d s \nabla G_{t-s} \cdot u_{s} \frac{\partial \boldsymbol{\omega}_{s}}{\partial y}
$$


by which

$$
\begin{aligned}
\left\|\frac{\partial}{\partial y} \omega_{t}\right\| \leqq & \left\|\omega_{0}\right\|^{(0,1)}+\left\|\mathscr{H}_{t}\right\|^{(0,1)} \\
& +\int_{0}^{t} d s \frac{1}{\sqrt{t-s}}\left\|u_{s}\right\|^{(0,1)}\left\|\omega_{s}\right\|+\int_{0}^{t} \frac{d s}{\sqrt{t-s}}\left\|u_{s}\right\|\left\|\frac{\partial \boldsymbol{\omega}_{s}}{\partial y}\right\|,
\end{aligned}
$$

suddenly implying $\omega \in \mathscr{B}_{T}^{(0,1)}$, because of Lemmas 4.1 and 4.5 and the fact that $u \in \overline{\mathscr{B}}_{T}^{(0,2)}$ as follows by (2.49) and (2.51). Finally, by (4.16), since $\|\nabla \boldsymbol{\omega}\|_{T}<\infty, \frac{\partial u}{\partial y}$ is a divergenceless vector field and $\frac{\partial^{2} u_{s}}{\partial y^{2}}$ is continuous in $y=0$ (it is the derivative of an odd function). Moreover,

$$
\begin{aligned}
\frac{\partial^{2}}{\partial y^{2}} \boldsymbol{\omega}_{t}= & G_{t}\left(\frac{\partial^{2} \boldsymbol{\omega}_{0}}{\partial y^{2}}+2 S^{\boldsymbol{\omega}_{0}} \boldsymbol{\delta}\right)+\frac{\partial^{2}}{\partial y^{2}} \mathscr{H}_{t} \\
& +\int_{0}^{t} d s G_{t-s} \cdot\left(\frac{\partial^{2} u_{s}}{\partial y^{2}}+2 S^{u_{s}} \boldsymbol{\delta}\right) \nabla \boldsymbol{\omega}_{0} \\
& +2 \int_{0}^{t} d s \nabla G_{t-s} \cdot \frac{\partial u_{s}}{\partial y} \frac{\partial \boldsymbol{\omega}_{s}}{\partial y}+\int_{0}^{t} d s \nabla G_{t-s} \cdot u_{s} \frac{\partial^{2} \boldsymbol{\omega}_{s}}{\partial y^{2}},
\end{aligned}
$$

where

$$
\begin{aligned}
& \left(S^{\boldsymbol{\omega}_{0}} \boldsymbol{\delta}\right)(x, y)=\left\{\frac{\partial \boldsymbol{\omega}_{0}}{\partial y}\left(x, 0^{+}\right)-\frac{\partial \boldsymbol{\omega}_{0}}{\partial y}\left(x, 0^{-}\right)\right\} \boldsymbol{\delta}(y), \\
& \left(S^{u_{s}} \boldsymbol{\delta}\right)(x, y)=\left\{\frac{\partial u_{s}}{\partial y}\left(x, 0^{+}\right)-\frac{\partial u_{s}}{\partial y}\left(x, 0^{-}\right)\right\} \boldsymbol{\delta}(y) .
\end{aligned}
$$

We can write again an integral inequality for $\left\|\frac{\partial^{2} \omega_{t}}{\partial y^{2}}\right\|$ implying $\sqrt{t} \frac{\partial^{2} \omega_{t}}{\partial y^{2}} \in \mathscr{B}_{T}$ for Lemmas 4.1 and 4.5 .

It remains to estimate the time derivatives. By (2.49) and (2.51), we have:

$$
\left\|\partial_{t} u_{t}^{1}\right\| \leqq c\left\|f_{t}\right\|+c\left\|u_{t}\right\|\left\|\omega_{t}\right\|+c\left(\left\|\omega_{t}\right\|^{(1,0)}+\left\|\omega_{t}\right\|^{(0,1)}\right),
$$

and an analogous estimate for $\partial_{t} u_{t}^{2}$.

Finally, the estimate on $\partial_{t} \omega$ follows by the estimate on $\partial_{y y}^{2} \omega$, the previous estimates and the structure of the equation for $\boldsymbol{\omega}$.

\section{Concluding Remarks}

We conclude by discussing some cases of physical interest, not explicitly treated in our previous analysis.

If a sufficiently smooth external field $g$ such that $\operatorname{curl} g \neq 0$ is acting on the system, all our considerations remain valid just replacing $F_{t}$ by $F_{t}+\operatorname{curl} g_{t}$. It is well known $[1,2]$ that the a priori estimates we make use of in Sect. 3 still hold under suitable hypotheses on $g$. Moreover, it is a standard fact that the hypotheses on the 
initial profile of velocity, $u_{0}(z) \rightarrow 0$ as $|z| \rightarrow \infty$, can be removed. In fact, by putting $w=u-u_{0}$ the Navier-Stokes equations reduces to

$$
\left\{\begin{array}{l}
\frac{\partial}{\partial t} w_{t}+w_{t} \cdot \nabla w_{t}+u_{0} \cdot \nabla w_{t}+w_{t} \cdot \nabla u_{0}+\nabla p=\Delta w_{t}+g+v \Delta u_{0}-u_{0} \nabla u_{0}, \\
\nabla \cdot w_{t}=0, \quad w_{0}=0, \quad w_{t}\left(x, 0^{+}\right)=0 .
\end{array}\right.
$$

It is easy to realize that, under suitable hypotheses on $u_{0}$, the above problem can be treated along the same lines of the one we have considered, the two systems differing only for trivial linear terms.

The case in which one needs to consider non-homogeneous boundary conditions like $u_{t}^{2}\left(x, 0^{+}\right)=0, u_{t}^{1}\left(x, 0^{+}\right)=a$, can be analyzed by subtracting to $u$ a time independent vector field $b$ such that $b^{2}=0, b^{1}=0$, for $y=0$. The resulting equations for $w_{t}=u_{t}-b$ admit homogeneous boundary conditions and differs from the previous one only by linear terms.

Finally, let us consider the case in which $u_{0}^{2}=0, u_{0}^{1} \neq 0$ for $y=0$, and we look for solutions for which the usual conditions, $u=0$ for $y=0$, are satisfied. For the Stokes problem the same analysis of Sect. 1 can be carried out, yielding Eqs. (1.16)-(1.18). Putting

$$
f_{t}(x)=\tilde{f}_{t}(x)+\boldsymbol{\delta}(t) a(x),
$$

one can determine $a$ under the condition that $\tilde{f}_{t}$ is continuous in time, obtaining

$$
\frac{\partial}{\partial x} \Delta^{-1} \omega_{0}\left(x, 0^{+}\right)=\lim _{t \rightarrow 0^{+}} h_{t}(x)=\frac{\partial}{\partial x} \Delta^{-1} a \delta\left(x, 0^{+}\right) .
$$

Putting

$$
\tilde{h_{t}}(x)=\frac{\partial}{\partial x} \Delta^{-1} G_{t}\left(\boldsymbol{\omega}_{0}-a \boldsymbol{\delta}\right)(x, 0)
$$

Eq. (1.16) can be rewritten as

$$
\tilde{h}=\mathscr{L} \tilde{f}
$$

and $\tilde{h}$ satisfies $\lim _{t \rightarrow 0^{+}} \tilde{h_{t}}(x)=0$.

The presence of a vortex sheet $a \boldsymbol{\delta}$ in the initial profile of vorticity $\boldsymbol{\omega}_{0}$ does not perturb our analysis in an essential way. Its presence is reasonable to impose a jump discontinuity of the Neumann velocity in the normal component, in order to force the system (although in a singular way) to satisfy the right boundary conditions. Under suitable assumptions on $a$ (and hence on $\left.u_{0}\right) \mathscr{L}^{-1} \tilde{h}$ makes sense to give rise to a solution of the Stokes ibvp as in Sect. 1. The Navier-Stokes evolution can be constructed as in Sects. 2 and 3 by replacing $h$ by $h-\frac{\partial}{\partial x} \Delta^{-1} a \delta$.

\section{Appendix A}

Let $f: \mathbb{R}^{1} \times[0, T] \rightarrow \mathbb{R}^{1}$ be an infinitely differentiable function of compact support. Consider

$$
V_{t}(x, y)=\int_{0}^{t} d s G_{t-s} f_{s} \boldsymbol{\delta}(x, y)
$$


It is easy to show that $V_{t} \in L_{\infty} \cap L_{1}\left(\mathbb{R}^{2}\right), \forall t \in[0, T]$. Then Eq. (1.18) can be written in the following way,

$$
\begin{aligned}
(\mathscr{L} f)_{t}(x) & =\frac{1}{\pi} \int_{0}^{\infty} d y \int_{-\infty}^{+\infty} d x_{1} \frac{x-x_{1}}{\left(x-x_{1}\right)^{2}+y^{2}} V_{t}\left(x_{1}, y\right) \\
& =\lim _{R \rightarrow \infty} \frac{1}{\pi} \int_{1 \backslash R}^{R} d y \int_{-R+x}^{R+x} \frac{\left(x-x_{1}\right)}{\left(x-x_{1}\right)^{2}+y^{2}} V_{t}\left(x_{1}, y\right) .
\end{aligned}
$$

From (A.1)

$$
V_{t}\left(x_{1}, y\right)=\int_{0}^{t} d s g_{t-s}(y) \frac{1}{\sqrt{2 \pi}} \int d k e^{i k x_{1}} e^{-(t-s) k^{2}} \hat{f}_{s}(k),
$$

where $g$ is the one-dimensional heat kernel. Then, by Fubini's theorem:

$$
(\mathscr{L} f)_{t}(x)=\lim _{R \rightarrow \infty} \frac{1}{\sqrt{2 \pi}} \int d k e^{i k x} \int_{0}^{t} d s \hat{f}_{s}(k) e^{-(t-s) k^{2}} \boldsymbol{\theta}_{R}(k, t-s),
$$

where

$$
\boldsymbol{\theta}_{R}(k, t)=-\frac{1}{\pi} \int_{1 / R}^{R / 2} d y g_{t}(y) \int_{-R}^{R} d u e^{i k u} \frac{u}{u^{2}+y^{2}} .
$$

It is easy to show that

$$
\begin{gathered}
\left|\boldsymbol{\theta}_{R}(k, t)\right| \leqq c, \\
\lim _{\boldsymbol{R} \rightarrow \infty} \boldsymbol{\theta}_{\boldsymbol{R}}(k, t)=-i \boldsymbol{\varepsilon}(k) e^{t k^{2}} \boldsymbol{\varphi}\left(\sqrt{t k^{2}}\right),
\end{gathered}
$$

where

$$
\boldsymbol{\varphi}(x)=\frac{1}{\sqrt{\pi}} \int_{x}^{\infty} d x_{1} e^{-x_{1}^{2}} .
$$

By the Lebesgue dominated convergence theorem, we have

$$
\begin{gathered}
(\mathscr{L} f)_{t}(x)=\frac{1}{\sqrt{2 \pi}} \int d k \hat{h}_{t}(k) e^{i k x}, \\
\hat{h}_{t}(k)=-i \boldsymbol{\varepsilon}(k) \int_{0}^{t} d s \hat{f}_{s}(k) \varphi\left(\sqrt{(t-s)) k^{2}}\right) .
\end{gathered}
$$

Equation (A.10) suddenly implies that $\mathscr{L}$ is a bounded linear operator on a dense set of $\mathscr{B}_{1, T, \varepsilon}$, and hence can be extended as a continuous operator on $\mathscr{B}_{1, T, \varepsilon}$ into itself, for any $\varepsilon \in[0,1)$.

Let us now extend arbitrarily $\hat{f}_{t}(k)$ for $t>T$, so that $f \in \mathscr{B}_{1, \infty, \varepsilon}$ and $\hat{f}_{t}(k)=0$ for $t \geqq 2 T$. We extend also $\hat{h}_{t}(k)$ so that Eq. (A.10) is valid for any $t \geqq 0$. We can then define the Laplace transforms $F(\omega, k)$ and $H(\omega, k)$, of $f$ and $h$, respectively, via the expression

$$
F(\boldsymbol{\omega}, k)=\int_{0}^{\infty} d t e^{-\boldsymbol{\omega} t} \hat{f}_{t}(k)
$$

(and the similar one for $H$ ) for any complex $\boldsymbol{\omega}$ such that $\operatorname{Re} \boldsymbol{\omega}>0$. 
Since the Laplace transform of $\boldsymbol{\varphi}$ is explicitly known, we can translate Eq. (A.10) in

$$
2 i \varepsilon(k) H(\omega, k)=\frac{F(\omega, k)}{\omega+k^{2}+|k| \sqrt{\omega+k^{2}}},
$$

which immediately implies that $\mathscr{L}$ is injective.

Let us now observe that Eq. (A.10) implies that, for any $f \in \mathscr{B}_{1, T, \varepsilon}, \hat{h}_{t}(k)$ is an absolutely continuous function of $t$, for a.a. $k$, and that $\hat{h}_{0}(k)=0$. Then

$$
\boldsymbol{\omega} H(k, \boldsymbol{\omega})=\int_{0}^{\infty} e^{-\boldsymbol{\omega} t} \frac{\partial \hat{h}_{t}}{\partial t}(k) d t
$$

Since

$$
\frac{1}{\sqrt{\omega+k^{2}}}=\int_{0}^{\infty} d t e^{-\omega t} \frac{1}{\sqrt{\pi t}} e^{-k^{2} t},
$$

Eq. (A.12) easily implies Eq. (1.21), if $h$ belongs to the range of $\mathscr{L}$. Finally, if $h$ satisfies conditions i) and ii) of the theorem, Eq. (A.13) is valid and we can invert the order of the previous calculations to show [provided that condition iii) is satisfied] that the function (1.21) is the solution of the equation $\mathscr{L} f=h$.

\section{Appendix B}

Lemma B.1. Consider the following integral

$$
\begin{aligned}
I_{\alpha_{1} \ldots \alpha_{n}}(t, s)= & \int_{s}^{t} \frac{d \tau_{1}}{\left(t-\tau_{1}\right)^{\alpha_{1}}} \int_{s}^{\tau_{1}} \frac{d \tau_{2}}{\left(\tau_{1}-\tau_{2}\right)^{\alpha_{2}}} \ldots \int_{s}^{\tau_{n-3}} \frac{d \tau_{n-2}}{\left(\tau_{n-s}-\tau_{n-2}\right)^{\alpha_{n-2}}} \\
& \cdot \int_{s}^{\tau_{n-2}} \frac{d \tau_{n-1}}{\left(\tau_{n-2}-\tau_{n-1}\right)^{\alpha_{n-1}}\left(\tau_{n-1}-s\right)^{\alpha_{n}}},
\end{aligned}
$$

where $\boldsymbol{\alpha}_{i}<1, i=1 \ldots n, t>s$. Then

Proof. Since

$$
I_{\alpha_{1} \ldots \alpha_{n}}(t, s)=(t-s)^{n-1-\sum_{i=1}^{n} \alpha_{i}} \frac{\prod_{i=1}^{n} \Gamma\left(1-\alpha_{i}\right)}{\Gamma\left(n-\sum_{i=1}^{n} \alpha_{i}\right)} .
$$

$$
\begin{aligned}
I_{\alpha_{1}, \alpha_{2}}(t, s) & =\int_{s}^{t} \frac{d \tau}{(t-\tau)^{\alpha_{1}}(\tau-s)^{\alpha_{2}}}=(t-s)^{1-\left(\alpha_{1}+\alpha_{2}\right)} \int_{0}^{1} \frac{d \xi}{(1-\xi)^{\alpha_{1} \xi^{\alpha_{2}}}} \\
& =(t-s)^{1-\left(\boldsymbol{\alpha}_{1}+\alpha_{2}\right)} \frac{\Gamma\left(1-\boldsymbol{\alpha}_{1}\right) \Gamma\left(1-\boldsymbol{\alpha}_{2}\right)}{\Gamma\left(2-\boldsymbol{\alpha}_{1}-\boldsymbol{\alpha}_{2}\right)}
\end{aligned}
$$

(B.2) follows by iteration.

Proof of Lemma 2.1. Defining the following operator

$$
(D \hat{\boldsymbol{\varphi}})(k, y)=\left(i k \hat{\boldsymbol{\varphi}}(k, y), \frac{\partial}{\partial y} \hat{\boldsymbol{\varphi}}(k, y)\right),
$$


we have

$$
\begin{aligned}
\left(V_{t, s}^{\wedge} \mathbf{\Phi}\right)(k, y)= & e^{-k^{2}(t-s)} \int g_{t-s}\left(y-y^{\prime}\right) \hat{\boldsymbol{\Phi}}\left(k, y^{\prime}\right) d y^{\prime} \\
& +\int_{s}^{t} d \tau e^{-k^{2}(t-\tau)} \int d y^{\prime} g_{t-\tau}\left(y-y^{\prime}\right) \int d h \hat{b}_{\tau}\left(k-h, y^{\prime}\right) \cdot D V_{\tau, s} \hat{\boldsymbol{\Phi}}\left(h, y^{\prime}\right) .
\end{aligned}
$$

Since $\nabla \cdot b_{t}=0$, by integrating by parts:

$$
\begin{aligned}
\left|V_{t, s}^{\wedge} \boldsymbol{\Phi}\right|(k, y) \leqq & \left.\sup _{y}|\boldsymbol{\Phi}(k, y)|+\sum_{i=1}^{2} \int_{s}^{t} d \tau e^{-k^{2}(t-\tau}\right) \\
& \cdot \int d y^{\prime} g_{t-\tau}\left(y-y^{\prime}\right)\left|\xi^{i}\left(\frac{y-y^{\prime}}{2(t-\tau)}, k\right)\right| \\
& \cdot \int d h\left|\hat{b}_{\tau}^{(i)}\left(k-h, y^{\prime}\right)\right|\left|V_{\tau, s}^{\wedge} \boldsymbol{\Phi}\right|\left(h, y^{\prime}\right),
\end{aligned}
$$

where

$$
\xi^{(i)}\left(\frac{y}{2 s}, k\right)=\left\{\begin{array}{llc}
i k & \text { if } & i=1 \\
\frac{y}{2 s} & \text { if } & i=2
\end{array}\right.
$$

then

$$
\left\|V_{t, s} \boldsymbol{\Phi}\right\| \leqq\|\boldsymbol{\Phi}\|+c\|b\|_{t} \int_{s}^{t} \frac{d \tau}{\sqrt{t-\tau}}\left\|V_{\tau, s} \boldsymbol{\Phi}\right\|
$$

By iteration:

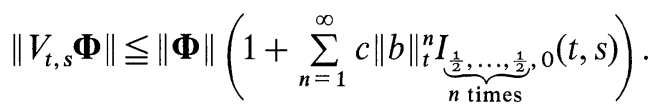

Finally, by virtue of Lemma B.1,

$$
\begin{aligned}
\left\|V_{t, s} \boldsymbol{\Phi}\right\| & \leqq\|\boldsymbol{\Phi}\|\left(1+\sum_{n=1}^{\infty}\left(c\|b\|_{t}\right)^{n}(t-s)^{n-\frac{n}{2}} \frac{\Gamma\left(\frac{1}{2}\right)^{n}}{\Gamma\left(n+1-\frac{n}{2}\right)}\right) \\
& \leqq\|\boldsymbol{\Phi}\|\left(\sum_{n \geqq 0} \frac{\left(c\|b\|_{t}\right)^{n}(t-s)^{n / 2}}{\Gamma\left(\frac{b}{2}+1\right)}\right) \\
& \leqq c\|\boldsymbol{\Phi}\| \exp c\|b\|_{t}^{2}(t-s) .
\end{aligned}
$$

If $\hat{\boldsymbol{\Phi}}(k, y)=\hat{\boldsymbol{\varphi}}(k) \boldsymbol{\delta}(y)$, we obtain

$$
y(t) \leqq c\|\varphi\|+c\|b\|_{t} \sqrt{t-s} \int_{s}^{t} \frac{d \tau}{\sqrt{t-\tau}} \frac{y(\tau)}{\sqrt{\tau-s}},
$$

where

$$
y(t)=\sqrt{t-s}\left\|V_{t, s} \boldsymbol{\Phi}\right\| .
$$


Hence

$$
y(t) \leqq c\|\boldsymbol{\varphi}\|(1+\sum_{n \geqq 1} c^{n}\|b\|_{t}^{n} \sqrt{t-s} \underbrace{I_{\frac{1}{2}}, \ldots, \frac{1}{2}}_{n \text { times }}(t, s)),
$$

implying (2.27) by Lemma B.1.

Furthermore, along the same lines,

$$
\begin{gathered}
\left\|\overline{\mathbf{\varrho}}_{t, s} \mathbf{\Phi}\right\| \leqq c\|b\|_{t}\|\mathbf{\Phi}\|+c\|b\|_{t} \int_{s}^{t} \frac{d \tau}{\sqrt{t-\tau}}\left\|\overline{\mathbf{\varrho}}_{\tau, s} \boldsymbol{\Phi}\right\|, \\
\left\|\mathbf{\varrho}_{t, s} \boldsymbol{\Phi}\right\| \leqq \frac{c\|b\|_{t}\|\mathbf{\Phi}\|}{\sqrt{t-s}}+c\|b\|_{t} \int_{s}^{t} \frac{d \tau}{\sqrt{t-\tau}}\left\|\mathbf{\varrho}_{\tau, s} \mathbf{\Phi}\right\|,
\end{gathered}
$$

and hence we obtain (2.25) and (2.26).

Finally, (2.28) and (2.29) are consequences of the extra divergence appearing in the first term of the right-hand side of (B.14) and (B.15) in case when $\boldsymbol{\Phi}=\boldsymbol{\varphi} \boldsymbol{\delta}$.

Proof of Lemma 2.2. By (2.37), recalling definition(B.4), integrating by parts, by the use of Lemma 2.1, we obtain

$$
\begin{aligned}
\hat{a}_{t}^{1}(k) & =\int d y D e^{-|k||y|} \cdot \int_{0}^{t} d s\left(\overline{\mathbf{\varrho}}_{t, s} F_{s}\right)(k, y), \\
\left\|a_{t}^{1}\right\| & \leqq \int_{0}^{t} d s\left\|\overline{\mathbf{\varrho}}_{t, s} F_{s}\right\| \sup _{k}\left(\int d y|k| e^{-|k||y|}\right) \\
& \leqq \int_{0}^{t} d s \frac{\left\|f_{s}\right\|}{\sqrt{t-s}}\left(c\|b\|_{t} \exp c t\|b\|_{t}^{2}\right) .
\end{aligned}
$$

Moreover, by (2.38)

$$
\begin{aligned}
&\left\|a_{t}^{2}\right\| \leqq \\
& \int_{0}^{t} d s \int_{s}^{t} \frac{d \tau}{\sqrt{t-\tau}} \int d k e^{-k^{2}(t-\tau)}|k| \\
& \cdot\left|\int d y \varepsilon(y) e^{-|k||y|} \int d y_{1} \frac{y-y_{1}}{2 \sqrt{t-\tau}} g_{t-\tau}\left(y-y_{1}\right)\left(\hat{\mathbf{Q}}_{\tau, s} F_{s}\right)\left(k, y_{1}\right)\right| \\
&= \int_{0}^{t} d s \int_{s}^{t} \frac{d \tau}{\sqrt{t-\tau}} J(t, \tau, s),
\end{aligned}
$$

where the last equality defines $J$.

We have

$$
J(t, \tau, s) \leqq \frac{c}{\sqrt{t-\tau}}\left\|\overline{\mathbf{\varrho}}_{\tau, s} F_{s}\right\| .
$$

In fact, proceeding as in the estimation of $\hat{a}^{1}$, we realize that the first component of $D$ gives rise to a factor $k$ and $\sup _{k} k^{2} e^{-k^{2}(t-\tau)} \int d y e^{-|k||y|} \leqq \frac{c}{\sqrt{t-\tau}}$. The second 
component, $i \frac{\partial}{\partial y_{1}}$, can be bounded in the same way because

$$
\int d y_{1}\left|\frac{\partial}{\partial y_{1}}\left(\frac{y-y_{1}}{2 \sqrt{t-\tau}} g_{t-\tau}\left(y-y_{1}\right)\right)\right| \leqq \frac{c}{\sqrt{t-\tau}} .
$$

On the other side, we have also

$$
J(t, \tau, s) \leqq c\left\|\boldsymbol{\varrho}_{\tau, s} F_{s}\right\| .
$$

Therefore, combining (B.19) and (B.20) and making use of (2.28) and (2.29),

$$
J(t, \tau, s) \leqq\left\{c\|b\|_{t} \exp \left(c t\|b\|_{t}^{2}\right)\right\} \frac{\left\|f_{s}\right\|}{(t-\tau)^{1 / 4}(\tau-s)^{3 / 4}} .
$$

We, finally, obtain (2.40) ${ }_{1}$ by inserting (B.21) and (B.18) and using (B.3).

To obtain $(2.40)_{2}$ we have

$$
\begin{aligned}
\left\|\left(S a^{1}\right)_{t}\right\| & \leqq \int_{0}^{t} \frac{d s}{\sqrt{t-s}} \int_{0}^{s} d \tau \int d k \frac{|k|}{\sqrt{\pi}} e^{-k^{2}(t-s)}\left|\int d y e^{-|k||y|}\left(\mathbf{Q}_{\hat{s}, \tau} F_{\tau}\right)(k, y)\right| \\
& =\int_{0}^{t} \frac{d s}{(t-s)^{1 / 2}} \int_{0}^{s} d \tau \int d k \frac{|k|}{\sqrt{\pi}} e^{-k^{2}(t-s)}|\widetilde{J}(s, \tau ; k)|,
\end{aligned}
$$

and the last equality defines $\tilde{J}$.

Two estimates are true

$$
\begin{gathered}
|\widetilde{J}| \leqq \frac{c}{|k|} \sup _{y}\left|\hat{\varrho}_{s, \tau} F_{\tau}\right|(k, y), \\
|\widetilde{J}| \leqq c \sup _{y}\left|\hat{\mathbf{\varrho}}_{s, \tau} F_{\tau}\right|(k, y),
\end{gathered}
$$

the second one again by integrating by parts. Hence

$$
\int d k|k| e^{-k^{2}(t-s)}|\widetilde{J}(s, \tau ; k)| \leqq c\left[\frac{\left\|\mathbf{\varrho}_{s, \tau} F_{\tau}\right\|\left\|\overline{\mathbf{\varrho}}_{s, \tau} F_{\tau}\right\|}{(t-s)^{1 / 2}}\right]^{1 / 2} .
$$

By virtue of (2.28) and (2.29),

$$
\left\|\left(S a^{1}\right)_{t}\right\| \leqq c\|b\|_{t} e^{c t\|b\|_{t}^{2}} \int_{0}^{t} \frac{d s}{(t-s)^{3 / 4}} \int_{0}^{s} \frac{d \tau}{(s-\tau)^{3 / 4}}\left\|f_{\tau}\right\|,
$$

implying $(2.40)_{2}$ for $i=1$, by interchanging integrations.

Finally,

$$
\begin{aligned}
\left\|\left(S a^{2}\right)_{t}\right\| \leqq & \frac{1}{\sqrt{\pi}} \int_{0}^{t} \frac{d s}{(t-s)^{1 / 2}} \int_{0}^{s} d \tau \int_{\tau}^{s} \frac{d \boldsymbol{\sigma}}{(s-\boldsymbol{\sigma})^{1 / 2}} \int d k k^{2} e^{-k^{2}(t-s)-k^{2}(s-\boldsymbol{\sigma})} \\
& \cdot\left|\int d y \varepsilon(y) e^{-|k||y|} \int d y_{1} \frac{\left(y-y_{1}\right)}{2(s-\boldsymbol{\sigma})^{1 / 2}} g_{s-\boldsymbol{\sigma}}\left(y-y_{1}\right)\left(\hat{\mathbf{Q}_{\boldsymbol{\sigma}}, \tau} F_{\tau}\right)\left(k, y_{1}\right)\right| .
\end{aligned}
$$


Denoting by $\bar{J}$ the quantity appearing in the modulus of the right-hand side of (B.27), we have

$$
\begin{aligned}
& |\bar{J}| \leqq c \sup _{y}||_{\hat{\boldsymbol{\sigma}}, \tau} F_{\tau} \mid(k, y), \\
& |\bar{J}| \leqq \frac{c}{|k|} \sup _{y}\left|\boldsymbol{\varrho}_{\hat{\boldsymbol{\sigma}}, \tau} F_{\tau}\right|(k, y) .
\end{aligned}
$$

Using as above both estimates (B.28) and (B.29), we have

$$
\left\|\left(S a^{2}\right)_{t}\right\| \leqq \int_{0}^{t} \frac{d s}{(t-s)^{7 / 8}} \int_{0}^{s} d \tau \int_{0}^{s} \frac{d \boldsymbol{\sigma}}{(s-\boldsymbol{\sigma})^{7 / 8}} \frac{1}{(\boldsymbol{\sigma}-\tau)^{3 / 4}}\left\|f_{\tau}\right\|\left(c\|b\|_{t} e^{e t\|b\|_{t}^{2}}\right)
$$

yielding $(2.40)_{2}, i=2$, after integrating.

Proof of Lemma 2.3. By the use of the same arguments leading to (2.41), (2.37), (2.38), we obtain

$$
\mathscr{L}^{-\hat{1}} h=z^{1}+z^{2}+s\left(z^{1}+z^{2}\right)
$$

where

$$
\begin{gathered}
z_{t}^{1}(k)=-\int d y e^{-|k||y|}\left(\mathbf{\varrho}_{t, 0} \boldsymbol{\omega}_{0}\right)(k, y), \\
z_{t}^{2}(k)=|k| \int d y \varepsilon(y) e^{-|k||y|}\left\{\frac{e^{-k^{2} t}}{\sqrt{t}} \int d y_{1} \frac{y-y_{1}}{2 \sqrt{t}} g_{t}\left(y-y_{1}\right) \hat{\boldsymbol{\omega}}_{0}\left(k, y_{1}\right)\right\} \\
+\int_{0}^{t} \frac{d \tau}{\sqrt{t-\tau}} e^{-k^{2}(t-\tau)} \int d y_{1} \frac{y-y_{1}}{2 \sqrt{t-\tau}} g_{t-\tau}\left(y-y_{1}\right)\left(\varrho_{\tau, 0} \hat{\boldsymbol{\omega}}_{0}\right)\left(k, y_{1}\right) .
\end{gathered}
$$

Proceeding as in the proof of Lemma 2.2, we obtain

$$
\begin{gathered}
\left\|z_{t}^{1}\right\| \leqq c\left\|\overline{\mathbf{\varrho}}_{t, 0} \boldsymbol{\omega}_{0}\right\|, \\
\left\|z_{t}^{2}\right\| \leqq \frac{c\left\|\boldsymbol{\omega}_{0}\right\|}{\sqrt{t}}+c \int_{0}^{t} \frac{d \tau}{\sqrt{t-\tau}}\left\|\boldsymbol{\varrho}_{\tau, 0} \boldsymbol{\omega}_{0}\right\|, \\
\left\|\left(S z^{1}\right)_{t}\right\| \leqq c \int_{0}^{t} \frac{d \tau}{\sqrt{t-\tau}}\left\|\boldsymbol{\varrho}_{\tau, 0} \boldsymbol{\omega}_{0}\right\|, \\
\left\|\left(S z^{2}\right)_{t}\right\| \leqq\left\|\boldsymbol{\omega}_{0}\right\| \int_{0}^{t} \frac{d \tau}{(t-\tau)^{3 / 4} \tau^{3 / 4}}+c \int_{0}^{t} \frac{d \tau}{(t-\tau)^{3 / 4}} \int_{0}^{\tau} \frac{d \boldsymbol{\sigma}}{(\tau-\boldsymbol{\sigma})^{3 / 4}}\left\|\boldsymbol{\varrho}_{\boldsymbol{\sigma}, 0} \boldsymbol{\omega}_{0}\right\| .
\end{gathered}
$$

This achieves the proof by the use of Lemma 2.1 .

Proof of Lemma 2.4. Writing

$$
\boldsymbol{\omega}_{t}=G_{t} \boldsymbol{\omega}_{0}+\int_{0}^{t} d s G_{t-s} b_{s} \cdot \nabla V_{s, 0} \boldsymbol{\omega}_{0}+\int_{0}^{t} d s V_{t, s} F_{s},
$$

and inserting it in the expression of $\hat{v}^{(1)}$ [see (2.49)], we obtain $\hat{v}^{(1)}$ as a sum of three terms, the first of which is bounded in the $\|\cdot\|$ norm by $\left\|\hat{v}_{0}\right\|$ since $\nabla^{\perp} \Delta^{-1} G_{t} \omega_{0}$ $=G_{t} \nabla^{\perp} \Delta^{-1} \boldsymbol{\omega}_{0}$ and $\left\|G_{t} \boldsymbol{\Phi}\right\| \leqq\|\boldsymbol{\Phi}\|$. 
The second term is estimated in the following way

$$
\begin{aligned}
& \int_{0}^{t} d s \int d k \sup _{y}\left|\int d y_{1} \int d y_{2} e^{-|k|\left|y-y_{1}\right|} \frac{\boldsymbol{\varepsilon}}{2}\left(y-y_{1}\right) g_{t-s}\left(y_{1}-y_{2}\right) e^{-k^{2}(t-s)} D \overline{\mathbf{Q}}_{s, 0} \hat{\mathbf{\omega}}_{0}\left(k, y_{2}\right)\right| \\
& \quad \leqq c \int_{0}^{t} d s\left\|\overline{\mathbf{Q}}_{s, 0} \boldsymbol{\omega}_{0}\right\| .
\end{aligned}
$$

Finally, for the last term we have

$$
\begin{aligned}
& \int_{0}^{t} d s \int d k \sup _{y}\left\{\left|\int d y_{1} e^{-|k|\left|y_{1}-y\right|} \frac{\boldsymbol{\varepsilon}}{2}\left(y-y_{1}\right) g_{t-s}\left(y_{1}\right) e^{-k^{2}(t-s)} \hat{f}_{s}(k)\right|\right\} \\
& +\mid \int_{0}^{t} d s \int_{0}^{s} d \tau \int d y_{1} \int d y_{2} e^{-|k|\left|y_{1}-y\right|} \\
& \quad \cdot \frac{\boldsymbol{\varepsilon}}{2}\left(y-y_{1}\right) g_{t-s}\left(y_{1}-y_{2}\right) e^{-k^{2}(t-s)} D \cdot \overline{\mathbf{Q}}_{\tau, s} F_{s}\left(k, y_{2}\right) \mid .
\end{aligned}
$$

Proceeding as above and making use of an analogous estimate for $\hat{v}_{2}$, we obtain

$$
\left\|v_{t}\right\| \leqq\left\|v_{0}\right\|+c \int_{0}^{t} d s\left\|\overline{\mathbf{\varrho}}_{s, 0} \boldsymbol{\omega}_{0}\right\|+\frac{1}{2} \int_{0}^{t} d s\left\|f_{s}\right\|+c \int_{0}^{t} d s \int_{s}^{t} d \tau\left\|\overline{\mathbf{\varrho}}_{\tau, s} F_{s}\right\| .
$$

Estimate (2.53) can be obtained by Lemma 2.1 and Theorem 2.1, thanks to Eq. (2.52).

To achieve the proof of the lemma it is enough to prove that $\left\|\partial_{x} v_{t}\right\|$ and $\left\|\partial_{y} v_{t}\right\|$ are finite, the rest of the properties being evident.

In fact,

$$
\int d k \sup _{y}\left|i k \hat{v}_{t}\right|(k, y) \leqq c \int d k \int d y_{1}|k| e^{-|k|\left|y_{1}-y\right|} \sup _{y_{1}}\left|\hat{\boldsymbol{\omega}}_{t}\left(k, y_{1}\right)\right| \leqq\left\|\boldsymbol{\omega}_{t}\right\| .
$$

Furthermore,

$$
\begin{aligned}
\frac{\partial}{\partial y} \hat{v}_{t}^{1}(k, y)= & \frac{1}{2} \frac{\partial}{\partial y}\left\{\int_{y}^{+\infty} d y_{1} \hat{\boldsymbol{\omega}}_{t}\left(k, y_{1}\right) e^{-|k|\left(y_{1}-y\right)}-\int_{-\infty}^{y} d y_{1} \hat{\boldsymbol{\omega}}_{t}\left(k, y_{1}\right) e^{-|k|\left(y_{1}-y\right)}\right\} \\
= & -\hat{\boldsymbol{\omega}}_{t}(k, y)+\frac{1}{2} \int_{-\infty}^{+\infty} d y_{1} \hat{\boldsymbol{\omega}}_{t}\left(k, y_{1}\right)|k| e^{-|k|\left(y_{1}-y\right)} \\
& \frac{\partial}{\partial y} \hat{v}_{t}^{2}(k, y)=\frac{1}{2 i} k \int d y_{1} \hat{\boldsymbol{\omega}}_{t}\left(k, y_{1}\right) \boldsymbol{\varepsilon}\left(y_{1}-y\right) e^{-|k|\left(y_{1}-y\right)}
\end{aligned}
$$

Therefore,

$$
\max _{i=1,2} \int d k \sup _{y}\left|\frac{\partial \hat{v}^{(i)}}{\partial y}\right|(k, y) \leqq c\left\|\boldsymbol{\omega}_{t}\right\| .
$$

The thesis follows since $\boldsymbol{\omega} \in \mathscr{B}_{T}$. 


\section{Appendix C}

Proof of Proposition 3.1. By (3.5)

$$
\begin{aligned}
\left|\left(\overline{\mathbf{Q}}_{t, 0}^{(\hat{1})}-\overline{\mathbf{Q}}_{t, 0}^{(2)}\right) \boldsymbol{\omega}_{0}(k, y)\right| \leqq & \int d h \sup _{y}\left|\hat{b}_{t}^{(1)}-\hat{b}_{t}^{(2)}\right|(k-h, y) \sup _{y}\left|\hat{\boldsymbol{\omega}}_{0}(h, y)\right| \\
& +\int_{0}^{t} d \tau\left|\int d h\left(\hat{b}_{t}^{(1)}-\hat{b}_{t}^{(2)}\right)(k-h, y) G_{t-\tau} \mathbf{\varrho}_{\tau, 0}^{(\hat{1})} \boldsymbol{\omega}_{0}(k, y)\right| \\
& +\int_{0}^{t} d \tau\left|\int d h \hat{b}_{t}^{(2)}(k-h, y) G_{t-\tau}\left(\mathbf{Q}_{\tau, 0}^{(1)}-\mathbf{Q}_{\tau, 0}^{(\hat{2})}\right) \boldsymbol{\omega}_{0}(k, y)\right| .
\end{aligned}
$$

Proceeding as in the proof of Lemma 2.1,

$$
\begin{aligned}
\left\|\left(\overline{\mathbf{\varrho}}_{t, 0}^{(1)}-\overline{\mathbf{\varrho}}_{t, 0}^{(2)}\right) \boldsymbol{\omega}_{0}\right\| \leqq & \left\|b_{t}^{(1)}-b_{t}^{(2)}\right\|\left\|\boldsymbol{\omega}_{0}\right\|+c\left\|b_{1}^{(1)}-b_{2}^{(2)}\right\|_{t} \int_{0}^{t} d \tau \frac{1}{\sqrt{t-\tau}}\left\|\overline{\mathbf{\varrho}}_{\tau, 0}^{(1)} \boldsymbol{\omega}_{0}\right\| \\
& +c b_{T} \int_{0}^{t} d \tau \frac{1}{\sqrt{t-\tau}}\left\|\left(\overline{\mathbf{\varrho}}_{\tau, 0}^{(1)}-\overline{\mathbf{\varrho}}_{\tau, 0}^{(2)}\right) \boldsymbol{\omega}_{0}\right\| .
\end{aligned}
$$

By the use of $(2.25)$

$$
\begin{aligned}
\left\|\left(\overline{\mathbf{\varrho}}_{t, 0}^{(1)}-\overline{\mathbf{\varrho}}_{t, 0}^{(2)}\right) \boldsymbol{\omega}_{0}\right\| \leqq & c\left\|b_{t}^{(1)}-b_{t}^{(2)}\right\|\left\|\boldsymbol{\omega}_{0}\right\|\left(1+b_{T} e^{c t b_{T}^{2}} \sqrt{t}\right) \\
& +c b_{T} \int_{0}^{t} d \tau \frac{1}{\sqrt{t-\tau}}\left\|\left(\overline{\mathbf{\varrho}}_{\tau, 0}^{(1)}-\overline{\mathbf{\varrho}}_{\tau, 0}^{(2)}\right) \boldsymbol{\omega}_{0}\right\| .
\end{aligned}
$$

The thesis follows by iterating the inequality as in Lemma 2.1.

Estimate (3.4) can be obtained along the same lines.

To prove Estimatte (3.2) we write

$$
\begin{aligned}
f^{(1)}-f^{(2)}= & \left(1-\mathscr{T}^{(1)}\right)^{-1}\left(\mathscr{L}^{-1} h^{(1)}-\mathscr{L}^{-1} h^{(2)}\right) \\
& +\left[\left(1-\mathscr{T}^{(1)}\right)^{-1}-\left(1-\mathscr{T}^{(2)}\right)^{-1}\right] \mathscr{L}^{-1} h^{(2)} .
\end{aligned}
$$

By Theorem 2.1 we have

$$
\left\|\left(1-\mathscr{T}^{(1)}\right)^{-1} l\right\| \leqq \tilde{\gamma}(t)\|l\| .
$$

Furthermore, $\mathscr{L}^{-1} h^{(1)}-\mathscr{L}^{-1} h^{(2)}$ can be evaluated as in Appendix B, proof of Lemma 3.3, as a sum of four terms in which appears the difference $\left(\varrho_{\sigma, 0}^{(1)}-\varrho_{\sigma, 0}^{(2)}\right) \boldsymbol{\omega}_{0}$. By using Estimate (3.3), we, finally, get

$$
\left\|\left(\mathscr{L}^{-1} h^{(1)}-\mathscr{L}^{-1} h^{(2)}\right)_{t}\right\| \leqq c\left\|b^{(1)}-b^{(2)}\right\|_{t} e^{c t b_{T}^{2}}\left\|\omega_{0}\right\|
$$

(the most singular term disappears by difference).

It remains to estimate the $2^{\text {nd }}$ term in the right-hand side of (C.4). By expanding in a series,

$$
\begin{aligned}
& \left\|\left(\left[\left(1-\mathscr{T}^{(2)}\right)^{-1}-\left(1-\mathscr{T}^{(1)}\right)^{-1}\right] \mathscr{L}^{-1} h^{(2)}\right)_{t}\right\| \\
& \quad \leqq \sum_{n=1}^{\infty}\left\|\left(\left[\mathscr{T}^{(2) n}-\mathscr{T}^{(1) n}\right] \mathscr{L}^{-1} h^{(2)}\right)_{t}\right\|
\end{aligned}
$$


Proceeding as in the proof of Lemma 2.2, Appendix B we can write $\left(\mathscr{T}^{(1)}-\mathscr{T}^{(2)}\right) f^{(2)}$ as a sum of four terms similar to $a^{1}+a^{2}+S\left(a^{1}+a^{2}\right)$ [see (2.37)-(2.39)] in which $\varrho_{\tau, s} F_{s}$ and $\bar{\varrho}_{\tau, s} F_{s}$ are replaced by $\left(\varrho_{\tau, s}^{(1)}-\varrho_{\tau, s}^{(2)} F_{s}^{(2)}\right.$ and $\left(\bar{\varrho}_{\tau, s}^{(1)}-\overline{\boldsymbol{\varrho}}_{\tau, s}^{(2)}\right) F_{s}^{(2)}$, where $F_{s}^{(2)}=f_{s}^{(2)} \boldsymbol{\delta}$. Therefore,

$$
\left\|\left[\left(\mathscr{T}^{(1)}-\mathscr{T}^{(2)}\right) f\right]_{t}\right\| \leqq c\left\|b_{t}^{(1)}-b_{t}^{(2)}\right\| e^{c t b_{T}^{2}} \int_{0}^{t} \frac{d s}{\sqrt{t-s}}\left\|f_{s}\right\| .
$$

Hence (recalling that $\left\|\left(\mathscr{T}^{(i)} f\right)_{t}\right\| \leqq c e^{c b_{T}^{2} t} b_{T} \int_{0}^{t} \frac{d s}{\sqrt{t-s}}\left\|f_{s}\right\|$ by Lemma 2.2)

$$
\begin{aligned}
& \text { right-hand side of }(\mathrm{C} .7) \leqq \\
& \sum_{n=1}^{\infty} \sum_{k=0}^{n-1}\left\|\left[\mathscr{T}^{(1) k}\left(\mathscr{T}^{(1)}-\mathscr{T}^{(2)}\right) \mathscr{T}^{(2) n-k-1} \mathscr{L}^{-1} h^{(2)}\right]_{t}\right\| \\
& \leqq \sum_{n=1}^{\infty} n\left(c b_{T}\right)^{n-1} e^{n c b_{T}^{2} t}\left\|b^{(1)}-b^{(2)}\right\|_{t} \\
& \cdot \int_{0}^{t} d \tau_{1} \int_{0}^{\tau_{1}} d \tau_{2} \ldots \int_{0}^{\tau_{n}-1} d \tau_{n} \frac{1}{\sqrt{t-\tau_{1}}} \cdots \frac{1}{\sqrt{\tau_{n-1}-\tau_{n}}}\left\|\mathscr{L}^{-1} h^{(2)}\right\|_{\tau_{n}} .
\end{aligned}
$$

By virtue of Lemma B.1 and Lemma 2.3, we obtain (3.2).

Finally, using the same arguments leading to (B.41)

$$
\begin{aligned}
\left\|u_{t}^{(1)}-u_{t}^{(2)}\right\|= & \left\|v_{t}^{(1)}-v_{t}^{(2)}\right\| \leqq c \int_{0}^{t} d \tau\left\|\overline{\mathbf{\varrho}}_{\tau, 0}^{(1)} \boldsymbol{\omega}_{0}-\overline{\mathbf{\varrho}}_{\tau, 0}^{(2)} \boldsymbol{\omega}_{0}\right\| \\
& +\frac{1}{2} \int_{0}^{t} d s\left\|f_{s}^{(1)}-f_{s}^{(2)}\right\| \\
& +c \int_{0}^{t} d s \int_{s}^{t} d \tau\left\|\left(\overline{\mathbf{\varrho}}_{\tau, s}^{(1)}-\overline{\mathbf{\varrho}}_{\tau, s}^{(2)}\right) F_{s}^{(1)}\right\| \\
& +c \int_{0}^{t} d s \int_{s}^{t} d \tau\left\|\overline{\mathbf{\varrho}}_{\tau, s}^{(2)}\left(F_{s}^{(1)}-F_{s}^{(2)}\right)\right\| .
\end{aligned}
$$

The thesis follows by inserting Estimates (3.2)-(3.4).

Proof of Lemma 3.1. We prove the Lemma for $h^{+}$smooth. The general case is recovered by usual density arguments.

To solve the elliptic problem (3.37), we introduce a sheet of vorticity as in Sect. 1, to take into account the boundary conditions.

Let $\boldsymbol{\omega}$ be the even extension to $\mathbb{R}^{2}$ of $\omega^{+}=\operatorname{curl} u^{+}$. Then $\boldsymbol{\omega}$ solves the following problem

$$
(1-\Delta) \boldsymbol{\omega}=g+F,
$$

where $g$ is the even extension of $g^{+}=\operatorname{curl} h^{+}$,

$$
F(x, y)=f(x) \boldsymbol{\delta}(y)
$$

and $f$ has to be determined by the condition

$$
\left(\frac{\partial}{\partial x} \Delta^{-1} \boldsymbol{\omega}\right)(x, 0)=0 \text {. }
$$


Taking the Fourier transform of (C.11),

and (C.13) becomes

$$
\left(1+\xi^{2}\right) \tilde{\boldsymbol{\omega}}(\xi)=\tilde{g}(\xi)+\hat{f}(k) \cdot \frac{1}{\sqrt{2 \pi}}, \quad \xi=(k, q),
$$

$$
\int d q \frac{k}{k^{2}+q^{2}} \tilde{\boldsymbol{\omega}}(k, q)=0 .
$$

Inserting (C.14) in (C.15) and making use of the formula

we obtain

$$
\int \frac{d q}{\left(k^{2}+q^{2}\right)\left(1+k^{2}+q^{2}\right)}=\frac{\pi}{|k| \sqrt{1+k^{2}}\left(\sqrt{1+k^{2}}+|k|\right)},
$$

$$
\hat{f}(k)=-\sqrt{\frac{2}{\pi}}|k| \sqrt{1+k^{2}}\left(\sqrt{1+k^{2}}+|k|\right) \int d q^{\prime} \frac{\tilde{g}\left(k, q^{\prime}\right)}{\left(\mathrm{k}^{2}+\mathrm{q}^{\prime 2}\right)\left(1+\mathrm{k}^{2}+\mathrm{q}^{\prime 2}\right)} .
$$

On the other hand, if $h=\left(h^{1}, h^{2}\right)$, where $h^{1}$ is the odd extension of $h^{+1}$ and $h^{2}$ is the even extension of $h^{+2}$, we have

$$
\tilde{g}(k, q)=i k \tilde{h}^{2}(k, q)-i q \tilde{h}^{1}(k, q)+\frac{1}{\sqrt{2 \pi}} \lim _{y \rightarrow 0^{+}}\left[\tilde{h}^{1}(k, y)-\tilde{h}^{1}(k,-y)\right] .
$$

The term in square brackets of (C.18) gives no contribution in the expression of $\tilde{\omega}$, so that we can write

where

$$
\tilde{\boldsymbol{\omega}}(\xi)=\frac{i \xi^{\perp} \cdot \tilde{h}(\xi)+(2 \pi)^{-1} \hat{f}_{1}(k)}{1+\xi^{2}}
$$

$$
\begin{gathered}
\hat{f}_{1}(k)=-\sqrt{\frac{2}{\pi}}|k| \sqrt{1+k^{2}}\left(\sqrt{1+k^{2}}+|k|\right) \int d q^{\prime} \frac{i \xi^{\perp} \cdot \tilde{h}\left(\xi^{\prime}\right)}{\left(1+\xi^{\prime 2}\right) \xi^{\prime 2}} \\
{\left[\xi^{\prime}=\left(k, q^{\prime}\right)\right] .}
\end{gathered}
$$

Putting $v=\nabla^{\perp} \Delta^{-1} \omega$, we have

$$
\|\tilde{v}\|_{1} \leqq\left\|\tilde{v}_{1}\right\|_{1}+\left\|\tilde{v}_{2}\right\|_{1}
$$

where

$$
\tilde{v}_{1}(\xi)=-\frac{\xi^{\perp}}{\xi^{2}} \frac{\xi^{\perp} \cdot \tilde{h}(\xi)}{1+\xi^{2}}, \quad \tilde{v}_{2}(\xi)=\frac{i}{\sqrt{2 \pi}} \frac{\xi^{\perp}}{\xi^{2}} \frac{\hat{f}_{1}(k)}{1+\xi^{2}}
$$

Moreover, by the Hausdorff-Young theorem, $\tilde{h} \in L_{q}\left(\mathbb{R}^{2}\right)^{2}, q=\left(1-\frac{1}{p}\right)^{-1}$, and hence

$$
\begin{gathered}
\left\|\tilde{v}_{1}\right\|_{1} \leqq \int d \xi \frac{|\tilde{h}(\xi)|}{1+\xi^{2}} \leqq\left[\int d \xi \frac{1}{\left(1+\xi^{2}\right)^{p}}\right]^{1 / p} \cdot\|\tilde{h}\|_{q} \\
\left\|\tilde{v}_{2}\right\|_{1} \leqq c \int \frac{d k d q^{\prime}}{1+k^{2}+q^{\prime 2}}|\tilde{h}(\xi)| \sqrt{1+k^{2}}\left(\sqrt{1+k^{2}}+|k|\right) \\
\cdot \int d q \frac{|k|+|q|}{\left(k^{2}+q^{2}\right)\left(1+k^{2}+q^{2}\right)} .
\end{gathered}
$$


Using formulas (C.16) and

$$
\int d q \frac{|q|}{\left(k^{2}+q^{2}\right)\left(1+k^{2}+q^{2}\right)}=2 \log \left(1+\frac{1}{k^{2}}\right),
$$

we obtain

$$
\left\|\tilde{v}_{2}\right\|_{1} \leqq c \int d \xi \frac{|\log k|}{1+\xi^{2}}|\tilde{h}(\xi)| \leqq c\|\tilde{h}\|_{q} .
$$

The proof is achieved by the inequality $\|u\|=\|v\| \leqq\|\tilde{v}\|_{1}$.

\section{Appendix D}

Proof of Lemma 4.1. If $y>0$ :

$$
\hat{\mathscr{H}}_{t}(k, y)=\int_{0}^{t} d s e^{-k^{2}(t-s)} g_{t-s}(y) \hat{f}_{s}(k),
$$

then

$$
\begin{aligned}
\sup _{y>0}\left|k \partial_{y} \hat{\mathscr{H}}_{t}(k, y)\right|= & \sup _{y>0}\left|\int_{0}^{t} d s e^{-k^{2}(t-s)} k \frac{-y}{2(t-s)} \frac{e^{-y^{2} / 4(t-s)}}{\sqrt{4 \pi(t-s}} \hat{f}_{s}(k)\right| \\
\leqq & \left|k \hat{f}_{t}(k)\right| \sup _{y>0}\left[\frac{1}{\sqrt{4 \pi}} \int_{y / \sqrt{t}}^{\infty} d u \exp \left(-\frac{k^{2} y^{2}}{u^{2}}-\frac{u^{2}}{4}\right)\right] \\
& +c \int_{0}^{t} d s(t-s)^{-3 / 2}\left|\hat{f}_{t}(k)-\hat{f}_{s}(k)\right|,
\end{aligned}
$$

which implies Estimate (4.4). Moreover,

$$
\begin{aligned}
\sup _{y>0}\left|\partial_{y y} \hat{\mathscr{H}}_{t}(k, y)\right| & =\sup _{y>0}\left|\int_{0}^{t} \frac{d s}{2(t-s)} e^{-k^{2}(t-s)}\left[1-\frac{y^{2}}{2(t-s)}\right] g_{t-s}(x) \hat{f}_{s}(k)\right| \\
& \leqq\left|\hat{f}_{t}(k)\right| \sup _{y>0}\left|L_{t}(k, y)\right|+c \int_{0}^{t} \frac{d s}{(t-s)^{3 / 2}}\left|\hat{f}_{t}(k)-\hat{f}_{s}(k)\right|,
\end{aligned}
$$

where

$$
L_{t}(k, y)=\frac{1}{\sqrt{4 \pi} y} \int_{y / \sqrt{t}}^{\infty} d u\left(1-\frac{u^{2}}{2}\right) e^{-\frac{k^{2} y^{2}}{u^{2}}-\frac{u^{2}}{4}} .
$$

Since

$$
\int_{0}^{\infty} d u\left(1-\frac{u^{2}}{2}\right) e^{-u^{2} / 4}=0,
$$

we can write

$$
\begin{aligned}
L_{t}(k, y)= & -\frac{1}{\sqrt{4 \pi} y} \int_{0}^{y / \sqrt{t}} d u\left(1-\frac{u^{2}}{2}\right) e^{-u^{2} / 4} \\
& +\frac{1}{\sqrt{4} \pi y} \int_{y / \sqrt{t}}^{\infty} d u\left(1-\frac{u^{2}}{2}\right) e^{-u^{2} / 4}\left(e^{-\frac{k^{2} y^{2}}{u^{2}}}-1\right),
\end{aligned}
$$


which implies

$$
\begin{aligned}
\sup _{y>0}\left|L_{t}(k, y)\right| & \leqq \frac{c}{\sqrt{t}}+c|k| \sup _{y>0} \int_{1 / \sqrt{k^{2} t}}^{\infty} d \lambda e^{-\frac{k^{2} y^{2} \lambda^{2}}{8}}\left(1-e^{-1 / \lambda^{2}}\right) \\
& \leqq c\left(\frac{1}{\sqrt{t}}+|k|\right) . \quad \square
\end{aligned}
$$

Proof of Lemma 4.2. We shall use decomposition (B.31) of $\mathscr{L}^{-1} h$. From Eq. (B.32) it follows that

$$
\left\|z_{t}^{1}-z_{t}^{2}\right\|^{(0)} \leqq\left\|\overline{\mathbf{\varrho}}_{t, 0} \boldsymbol{\omega}_{0}-\overline{\mathbf{\varrho}}_{s, 0} \boldsymbol{\omega}_{0}\right\|^{(0,0)}
$$

By Eqs. (2.21), (2.22)

$$
\overline{\mathbf{\varrho}}_{t, s}=b_{t} G_{t-s}+\int_{0}^{t} d \tau b_{t} G_{t-\tau} \mathbf{\varrho}_{\tau, s},
$$

which implies $\left(\|\cdot\|=\|\cdot\|^{(0,0)}\right)$

$$
\begin{aligned}
\left\|\overline{\mathbf{\varrho}}_{t, 0} \boldsymbol{\omega}_{0}-\overline{\mathbf{\varrho}}_{s, 0} \boldsymbol{\omega}_{0}\right\| \leqq & \left\|\left(b_{t}-b_{s}\right) G_{t} \boldsymbol{\omega}_{0}\right\|+\left\|b_{s}\left(G_{t}-G_{s}\right) \boldsymbol{\omega}_{0}\right\| \\
& +\int_{s}^{t} d \tau\left\|b_{t} G_{t-\tau} \mathbf{\varrho}_{\tau, 0} \boldsymbol{\omega}_{0}\right\|+\int_{0}^{s} d \tau\left\|\left(b_{t}-b_{s}\right) G_{t-\tau} \mathbf{\varrho}_{\tau, 0} \boldsymbol{\omega}_{0}\right\| \\
& +\int_{0}^{s} d \tau\left\|b_{s}\left(G_{t-\tau}-G_{s-\tau}\right) \varrho_{\tau, 0} \boldsymbol{\omega}_{0}\right\| \\
\leqq & \left\|b_{t}-b_{s}\right\|\left\|\boldsymbol{\omega}_{0}+\right\| b_{s}\left\|\int_{s}^{t} d \boldsymbol{\sigma}\right\| \partial_{\boldsymbol{\sigma}} G_{\boldsymbol{\sigma}} \boldsymbol{\omega}_{0} \| \\
& +\left\|b_{t}\right\| \int_{s}^{t} d \tau\left\|\mathbf{\varrho}_{\tau, 0} \boldsymbol{\omega}_{0}\right\|+\left\|b_{t}-b_{s}\right\| \int_{0}^{s} d \tau\left\|\mathbf{\varrho}_{\tau, 0} \boldsymbol{\omega}_{0}\right\| \\
& +\left\|b_{s}\right\| \int_{0}^{s} d \tau \int_{s-\tau}^{t-\tau} d \boldsymbol{\sigma}\left\|\partial_{\sigma} G_{\sigma} \mathbf{\varrho}_{\tau, 0} \boldsymbol{\omega}_{0}\right\|
\end{aligned}
$$

We observe now that, if $\boldsymbol{\Phi} \in \mathscr{B}^{(1,0)} \cap \mathscr{B}^{(0,1)}$,

$$
\begin{aligned}
\left\|\partial_{\boldsymbol{\sigma}} G_{\boldsymbol{\sigma}} \boldsymbol{\Phi}\right\|^{(0,0)} & =\int d k \sup _{y}\left|\int d y_{1} \partial_{\boldsymbol{\sigma}}\left[e^{-k^{2} \boldsymbol{\sigma}} g_{\boldsymbol{\sigma}}\left(y-y_{1}\right)\right] \hat{\boldsymbol{\Phi}}\left(k, y_{1}\right)\right| \\
& \leqq \frac{c}{\sqrt{\boldsymbol{\sigma}}} \int d k\left[|k| \sup _{y}|\hat{\boldsymbol{\Phi}}(k, y)|+\sup _{y}\left|\partial_{y} \hat{\boldsymbol{\Phi}}(k, y)\right|\right] \\
& =\frac{c}{\sqrt{\boldsymbol{\sigma}}}\left(\|\boldsymbol{\Phi}\|^{(1,0)}+\|\boldsymbol{\Phi}\|^{(0,1)}\right),
\end{aligned}
$$

and we used the fact that, for $|y|>0$

$$
\partial_{\sigma} g_{\sigma}(y)=\partial_{y y} g_{\sigma}(y)
$$

and an integration by parts.

In order to use inequality (D.11), we need an estimate of $\left\|\boldsymbol{\varrho}_{\tau, 0} \boldsymbol{\omega}_{0}\right\|^{(1,0)}$ and $\left\|\boldsymbol{\varrho}_{\tau, 0} \boldsymbol{\omega}_{0}\right\|^{(0,1)}$, which can be obtained by an extension of the proof leading to Eq. (2.26) (see Appendix B). This is a very easy task and we obtain that for any $n$ and $m \leqq 1$

$$
\left\|\boldsymbol{\varrho}_{t, s} \boldsymbol{\Phi}_{s}\right\|^{(n, m)} \leqq \frac{c\|b\|_{t}^{(n, m)}}{\sqrt{t-s}} e^{c(t-s)\left(\|b\|_{t}^{(n, m))^{2}}\|\boldsymbol{\Phi}\|_{s}^{(n, m)}\right.}
$$

(the limitation on $m$ is due to possible discontinuities of the derivatives in $y=0$ ). 
Estimates (D.10, (D.11), (D.13) immediately imply that, if $\omega_{0} \in \mathscr{B}^{(1,0)} \cap \mathscr{B}^{(0,1)}$ and $b \in \overline{\mathscr{B}}_{T}^{(1,0)} \cap \overline{\mathscr{B}}^{(0,1)}$

$$
\begin{aligned}
\left\|\overline{\boldsymbol{\varrho}}_{t, 0} \boldsymbol{\omega}_{0}-\overline{\mathbf{\varrho}}_{s, 0} \boldsymbol{\omega}_{0}\right\|^{(0,0)} & \leqq c(T)\left[\left\|b_{t}-b_{s}\right\|^{(0,0)}+\int_{s}^{t} \frac{d \boldsymbol{\sigma}}{\sqrt{\boldsymbol{\sigma}}}+\int_{0}^{s} \frac{d \tau}{\sqrt{\tau}} \int_{s-\tau}^{t-\tau} \frac{d \boldsymbol{\sigma}}{\sqrt{\boldsymbol{\sigma}}}\right] \\
& \leqq c(T)\left[\left\|b_{t}-b_{s}\right\|^{(0,0)}+\frac{t-s}{\sqrt{s}}\right] .
\end{aligned}
$$

Let us now consider the function $z^{2}$ defined by (B.33). If $\omega_{0} \in \mathscr{B}^{(0,1)}$, we can write

$$
\begin{aligned}
z_{t}^{2}(k)= & |k| \int d y \boldsymbol{\varepsilon}(y) e^{-|k||y|}\left\{e^{-k^{2} t} \int d y_{1} g_{t}\left(y-y_{1}\right)\left(\partial_{y} \hat{\boldsymbol{\omega}}_{0}\right)\left(k, y_{1}\right)\right\} \\
& +\int_{0}^{t} d \tau e^{-k^{2}(t-\tau)} \int d y_{1} g_{t-\tau}\left(y-y_{1}\right)\left(\partial_{y} \hat{\mathbf{\varrho}}_{\tau, 0} \boldsymbol{\omega}_{0}\right)\left(k, y_{1}\right) .
\end{aligned}
$$

Proceeding as in the previous calculation, making an integration by parts in the left direction in the terms containing $\partial_{\sigma} g_{\sigma}$, we obtain

$$
\begin{aligned}
\left\|z_{t}^{2}-z_{s}^{2}\right\|^{(0)} \leqq & c \frac{t-s}{\sqrt{s}}\left\|\boldsymbol{\omega}_{0}\right\|^{(1,1)}+c \int_{s}^{t} d \tau\left\|\mathbf{\varrho}_{\tau, 0} \boldsymbol{\omega}_{0}\right\|^{(0,1)} \\
& +c \int_{0}^{s} d \tau \frac{t-s}{\sqrt{s-\tau}}\left\|\mathbf{\varrho}_{\tau, 0} \boldsymbol{\omega}_{0}\right\|^{(1,1)}
\end{aligned}
$$

Then, by using Eq. (D.13), we obtain that, if $\boldsymbol{\omega}_{0} \in \mathscr{B}^{(1,1)}$ and $b \in \overline{\mathscr{B}}_{T}^{(1,1)}$,

$$
\left\|z_{t}^{2}-z_{s}^{2}\right\|^{(0)} \leqq c(T) \frac{t-s}{\sqrt{s}} .
$$

We still have to study $\left\|(S z)_{t}-(S z)_{s}\right\|^{(0)}$, where $z=z^{1}+z^{2}$ and $S$ is the operator defined by Eq. (2.39). We can write

$$
(S \hat{z})_{t}(k)=\frac{|k|}{\sqrt{\pi}} \int_{0}^{t} \frac{d s}{\sqrt{s}} e^{-k^{2} s} \hat{z}_{t-s}(k)
$$

which implies

$$
\left\|(S z)_{t}-(S z)_{s}\right\|^{0} \leqq c \int_{s}^{t} \frac{d \tau}{\sqrt{\tau}}\left\|z_{t-\tau}\right\|^{(1)}+c \int_{0}^{s} \frac{d \tau}{\sqrt{\tau}}\left\|z_{t-\tau}-z_{s-\tau}\right\|^{(1)} .
$$

We note now that (2.25) can be easily generalized to obtain, for any $n$ and $m \leqq 1$

$$
\left\|\overline{\mathbf{Q}}_{t, s} \boldsymbol{\Phi}_{s}\right\|^{(n, m)} \leqq c\|b\|_{t}^{(n, m)} e^{c(t-s)\left(\|b\|_{t}^{(n, m)}\right)^{2}}\left\|\boldsymbol{\Phi}_{s}\right\|^{(n, m)} .
$$

Then, proceeding as in the proof of Lemma 2.3 (see Appendix B), we get, if $\omega_{0} \in \mathscr{B}^{(1,1)}$ and $b \in \overline{\mathscr{B}}_{T}^{(1,1)}$

$$
\begin{aligned}
\left\|z_{t}\right\|^{(1)} & \leqq c\left\|\overline{\mathbf{\varrho}}_{t, 0} \boldsymbol{\omega}_{0}\right\|^{(1,0)}+c\left\|\boldsymbol{\omega}_{0}\right\|^{(1,1)}+c \int_{0}^{t} \frac{d \tau}{\sqrt{t-\tau}}\left\|\mathbf{\varrho}_{\tau, 0} \boldsymbol{\omega}_{0}\right\|^{(1,0)} \\
& \leqq c(T) .
\end{aligned}
$$


Finally, we can repeat the steps leading to (D.14) and (D.17) in order to show that, if $\boldsymbol{\omega}_{0} \in \mathscr{B}^{(2,1)}$ and $b \in \overline{\mathscr{B}}_{T}^{(2,1)}$

$$
\left\|z_{t}-z_{s}\right\|^{(1)} \leqq c(T)\left[\left\|b_{t}-b_{s}\right\|^{(1,0)}+\frac{t-s}{\sqrt{s}}\right] .
$$

Estimates (D.19), (D.21), (D.22) imply that

$$
\left\|(S z)_{t}-(S z)_{s}\right\|^{(0)} \leqq c(T)\left[\frac{t-s}{\sqrt{s}}+\left\|b_{t}-b_{s}\right\|^{(1,0)}\right] .
$$

Hence (4.6) follows from (B.31), (D.14), (D.17), and (D.23).

Proof of Lemma 4.3. The solution $u$ of the Navier-Stokes equations is obtained by inserting (3.18) in the right-hand side of (2.49) and (2.51). To estimate $\left\|u_{t}-u_{s}\right\|^{(1,0)}$, we proceed as in the proof of Lemma 4.2, obtaining

$$
\begin{aligned}
\left\|u_{t}-u_{s}\right\|^{(1,0)} \leqq & c(t-s)\left[\left\|\boldsymbol{\omega}_{0}\right\|^{(2,0)}+\left\|\boldsymbol{\omega}_{0}\right\|^{(1,1)}\right]+c \int_{s}^{t} d \tau\left\|\overline{\mathbf{\varrho}}_{\tau, 0} \boldsymbol{\omega}_{0}\right\|^{(1,0)} \\
& +c \int_{0}^{s} d \tau \int_{s-\tau}^{t-\tau} \frac{d u}{u}\left\|\overline{\mathbf{\varrho}}_{\tau, 0} \boldsymbol{\omega}_{0}\right\|^{(1,0)}+c \int_{s}^{t} d \tau\left\|f_{\tau}\right\|^{(1)}+c \int_{0}^{s} d \tau \int_{s-\tau}^{t-\tau} \frac{d u}{u}\left\|f_{\tau}\right\|^{(1)} \\
& +c \int_{s}^{t} d \tau \int_{\tau}^{t} d \boldsymbol{\sigma}\left\|\overline{\mathbf{\varrho}}_{\boldsymbol{\sigma}}{ }_{\tau} F_{\tau}\right\|^{(1,0)}+c \int_{0}^{s} d \tau \int_{s}^{t} d \boldsymbol{\sigma}\left\|\overline{\mathbf{\varrho}}_{\boldsymbol{\sigma}, \tau} F_{\tau}\right\|^{(1,0)} \\
& +c \int_{0}^{s} d \tau \int_{\tau}^{s} d \boldsymbol{\sigma} \int_{s-\boldsymbol{\sigma}}^{t-\boldsymbol{\sigma}} \frac{d u}{u}\left\|\overline{\mathbf{\varrho}}_{\boldsymbol{\sigma}, \tau} F_{\tau}\right\|^{(1,0)} .
\end{aligned}
$$

An easy generalization of (2.28) gives, for all $n$

$$
\left\|\overline{\mathbf{Q}}_{\boldsymbol{\sigma}, \tau} F_{\tau}\right\|^{(n, 0)} \leqq c\|b\|_{\boldsymbol{\sigma}}^{(n)} \frac{\exp ^{c(\boldsymbol{\sigma}-\tau)\left(\|b\|^{(n, 0)}\right)^{2}}}{\sqrt{\boldsymbol{\sigma}-\tau}}\left\|f_{\tau}\right\|^{(n)} .
$$

Moreover, Theorem 2.1 can be extended to prove that, if $\omega_{0} \in \mathscr{B}^{(n, 1)}$ and $b \in \overline{\mathscr{B}}_{T}^{(n, 0)}$,

$$
\left\|f_{\tau}\right\|^{(n)} \leqq c(T) .
$$

In fact, proceeding as in the proof of Theorem 2.1, it is easy to obtain the analogue of Estimate (2.45), with $\left\|f_{t}\right\|$ replaced by $\left\|f_{t}\right\|^{(n)}$ and $\|b\|_{t},\left\|\omega_{0}\right\|$ by $\|b\|_{t}^{(n, 0)},\left\|\omega_{0}\right\|^{(n)}$. Finally, the $\frac{1}{\sqrt{t}}$ singularity [arising only by the bound (2.42)] can be removed by an extra integration by parts, involving the first $y$-derivative of $\omega_{0}$.

(D.20), (D.24)-(D.26), and (B.44) imply that, if $\omega_{0} \in \mathscr{B}^{(2,0)} \cap \mathscr{B}^{(1,1)}$ and $\varepsilon \in\left(0, \frac{1}{2}\right]$

$$
\begin{aligned}
\left\|u_{t}-u_{s}\right\|^{(1,0)} \leqq & c(T)\left[(t-s)+\int_{0}^{s} d \tau \int_{s-\tau}^{t-\tau} \frac{d u}{u}+\int_{0}^{s} d \tau \int_{s}^{t} \frac{d \boldsymbol{\sigma}}{\sqrt{\boldsymbol{\sigma}-\tau}}\right. \\
& \left.+\int_{0}^{s} d \tau \int_{\tau}^{s} \frac{d \boldsymbol{\sigma}}{\sqrt{\boldsymbol{\sigma}-\tau}} \int_{s-\sigma}^{t-\sigma} \frac{d u}{u}\right] \\
\leqq & c(T)\left[(s-t)+\int_{0}^{s} \frac{d \tau}{(s-\tau)^{1-\varepsilon}} \int_{s-\tau}^{t-\tau} \frac{d u}{u \varepsilon}+\int_{0}^{s} \frac{d \tau}{\sqrt{s-\tau}} \int_{s}^{t} d \boldsymbol{d \sigma}\right. \\
& \left.+\int_{0}^{s} d \tau \int_{\tau}^{s} \frac{d \boldsymbol{\sigma}}{\sqrt{\boldsymbol{\sigma}-\tau}(s-\boldsymbol{\sigma})^{1-\varepsilon}} \int_{s-\sigma}^{t-\sigma} \frac{d u}{u \varepsilon}\right] .
\end{aligned}
$$


(4.7) then follows by the inequality

$$
\int_{s-\tau}^{t-\tau} \frac{d u}{u \varepsilon} \leqq \int_{0}^{t-s} \frac{d u}{u \varepsilon}=\frac{(t-s)^{1-\varepsilon}}{1-\varepsilon} \leqq 2(t-s)^{1-\varepsilon} .
$$

Proof of Lemma 4.4. We shall use the decomposition (2.41) of $\mathscr{T} f$. Let us define

Equation (2.37) implies

$$
\|\boldsymbol{\Phi}\| \|^{(n)}=\int d y d k|\hat{\boldsymbol{\Phi}}(k, y)| \cdot|k|^{n} .
$$

$$
\begin{aligned}
\left\|a_{t}^{1}-a_{s}^{1}\right\|^{(0)} \leqq & \int_{s}^{t} d \tau\left\|\overline{\mathbf{Q}}_{t, \tau} F_{\tau}\right\| \|^{(1)} \\
& +\int_{0}^{s} d \tau \int d k\left|\int d y e^{-|k||y|}\left[\mathbf{\varrho}_{t, \tau} \widehat{F_{\tau}-\mathbf{Q}_{s, \tau}} F_{\tau}\right](k, y)\right| .
\end{aligned}
$$

Proceeding as in the proof of Lemma 2.1 , it is easy to show that, for all $n \geqq 0$

Moreover,

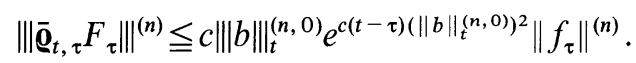

$$
\begin{aligned}
& \int d k\left|\int d y e^{-|k||y|}\left[\mathbf{\varrho}_{t, \tau} \widehat{F}_{\tau-\mathbf{Q}_{s, \tau}} F_{\tau}\right](k, y)\right| \\
& \leqq\left\|\left(b_{t}-b_{s}\right) G_{t-\tau} F_{\tau}\right\|^{(0,0)}+c \int_{s-\tau}^{t-\tau} d u\left\|b_{s} \partial_{u} G_{u} F_{\tau}\right\|^{(1)} \\
& +c \int_{0}^{t} d \boldsymbol{\sigma}\left\|b_{t} G_{t-\boldsymbol{\sigma}} \mathbf{\varrho}_{\boldsymbol{\sigma}, \tau} F_{\tau}\right\|^{(1)}+c \int_{\tau}^{s} d \boldsymbol{\sigma}\left\|\left(b_{t}-b_{s}\right) G_{t-\boldsymbol{\sigma}} \mathbf{\varrho}_{\boldsymbol{\sigma}, \tau} F_{\tau}\right\|^{(0,0)} \\
& +c \int_{\tau}^{s} d \boldsymbol{\sigma} \int_{s-\boldsymbol{\sigma}}^{t-\boldsymbol{\sigma}} d u\left\|b_{s} \partial_{u} G_{u} \mathbf{Q}_{\boldsymbol{\sigma}, \tau} F_{\tau}\right\| \|^{(1)} \\
& \leqq c\left\|b_{t}-b_{s}\right\|^{(0,0)} \frac{\left\|f_{\tau}\right\|^{(0)}}{\sqrt{s-\tau}}+c\|b\|_{t}^{(1,0)} \int_{s-\tau}^{t-\tau} \frac{d u}{u}\left\|f_{\tau}\right\|^{(1)} \\
& +c\left\|b_{t}\right\|^{(1,0)} \int_{s}^{t} d \boldsymbol{\sigma}\left\|\mathbf{\varrho}_{\boldsymbol{\sigma}, \tau} F_{\tau}\right\|^{(1)}+c\left\|b_{t}-b_{s}\right\|^{(0,0)} \int_{\tau}^{s} \frac{d \boldsymbol{\sigma}}{\sqrt{s-\boldsymbol{\sigma}}}\left\|\overline{\mathbf{\varrho}}_{\boldsymbol{\sigma}, \tau} F_{\tau}\right\|^{(0,0)} \\
& +c \int_{\tau}^{s} d \boldsymbol{\sigma} \int_{s-\boldsymbol{\sigma}}^{t-\boldsymbol{\sigma}} \frac{d u}{u}\|b\|_{t}^{(1,0)}\left\|\varrho_{\boldsymbol{\sigma}, \tau} F_{\tau}\right\|^{(1)},
\end{aligned}
$$

(2.28), (D.31) and the analogue

$$
\left\|\mathbf{\varrho}_{t, \tau} F_{\tau}\right\|^{(n)} \leqq c\|b\|_{t}^{(n, 0)} e^{c(t-\tau)\left(\|b\|_{t}^{(n, 0)}\right)^{2}} \frac{\left\|f_{\tau}\right\|^{(n)}}{\sqrt{t-\tau}}
$$

imply, if $b \in \overline{\mathscr{B}}_{T}^{(1,0)}$, for all $\boldsymbol{\varepsilon} \in\left(0, \frac{1}{2}\right]$

$$
\left\|a_{t}^{1}-a_{s}^{1}\right\|^{(0)} \leqq c(T)\left[(t-s)+\left\|b_{t}-b_{s}\right\|^{(0,0)}+\frac{(t-s)^{1-\varepsilon}}{\varepsilon}\right]\|f\|_{t}^{(1)} .
$$

Let us now consider the function $a_{t}$ defined by (2.38). We have

$$
\begin{aligned}
\left\|a_{t}^{2}-a_{s}^{2}\right\|^{(0)} \leqq & c \int_{s}^{t} d \boldsymbol{\sigma} \int_{\boldsymbol{\sigma}}^{t} \frac{d \tau}{(t-\tau)^{1 / 2}}\left\|\mathbf{\varrho}_{\tau, \boldsymbol{\sigma}} F_{\boldsymbol{\sigma}}\right\|^{(1)}+c \int_{0}^{s} d \boldsymbol{\sigma} \int_{s}^{t} d \tau\left\|\mathbf{\varrho}_{\tau, \boldsymbol{\sigma}} F_{\boldsymbol{\sigma}}\right\|^{(1,0)} \\
& +c \int_{0}^{s} d \boldsymbol{\sigma} \int_{\boldsymbol{\sigma}}^{s} d \tau \int_{s-\tau}^{t-\tau} d u\left[\frac{\left\|\mathbf{\varrho}_{\tau, \boldsymbol{\sigma}} F_{\boldsymbol{\sigma}}\right\|^{(1)}}{u^{3 / 2}}\right]^{\varepsilon}\left[\frac{\left\|\mathbf{\varrho}_{\tau, \boldsymbol{\sigma}} F_{\boldsymbol{\sigma}}\right\|^{(1,0)}}{u}\right]^{1-\boldsymbol{\varepsilon}}
\end{aligned}
$$


for any $\varepsilon \in\left(0, \frac{1}{2}\right]$. Therefore, by (2.29) and (D.33), if $b \in \overline{\mathscr{B}}_{T}^{(1,0)}$

$$
\begin{aligned}
\left\|a_{t}^{2}-a_{s}^{2}\right\|^{(0)} \leqq & c(T)\left[(t-s)+\int_{0}^{s} d \sigma \int_{s}^{t} \frac{d \tau}{\tau-\boldsymbol{\sigma}}\right. \\
& \left.+\int_{0}^{s} d \boldsymbol{\sigma} \int_{\boldsymbol{\sigma}}^{s} \frac{d \tau}{(\tau-\boldsymbol{\sigma})^{1-\varepsilon / 2}} \int_{s-\tau}^{t-\tau} \frac{d u}{u^{1+\varepsilon / 2}}\right]\|f\|_{t}^{(1)} \\
\leqq & c(T) \frac{(t-s)^{1-\varepsilon}}{\varepsilon}\|f\|_{t}^{(1)} .
\end{aligned}
$$

We have also by (D.19), if $a=a^{1}+a^{2}$

$$
\left\|(S a)_{t}-(S a)_{s}\right\|^{(0)} \leqq c \int_{s}^{t} \frac{d \tau}{\sqrt{\tau}}\left\|a_{t-\tau}\right\|^{(1)}+c \int_{0}^{s} \frac{d \tau}{\sqrt{\tau}}\left\|a_{t-\tau}-a_{s-\tau}\right\|^{(1)} .
$$

The same steps leading to (D.34) and (D.36) give also, changing the norm and supposing $b \in \overline{\mathscr{B}}_{T}^{(2,0)}$

$$
\left\|a_{t}-a_{s}\right\|^{(1)} \leqq c(T)\left[\left\|b_{t}-b_{s}\right\|^{(1,0)}+\frac{(t-s)^{1-\varepsilon}}{\varepsilon}\right]\|f\|_{t}^{(2)} .
$$

Moreover, if $b \in \overline{\mathscr{B}}_{T}^{(0,0)}$, by means of an easy generalization of $(2.41)_{1}$

$$
\left\|a_{t}\right\|^{(1)} \leqq c(T)\|f\|_{t}^{(1)} .
$$

The previous estimates immediately imply Estimate (4.8).

Proof of Lemma 4.5. (2.13), (4.6), and (4.8) imply

$$
\begin{aligned}
\left\|f_{t}-f_{s}\right\|^{(0)} \leqq & \left\|\left(\mathscr{L}^{-1} h\right)_{t}-\left(\mathscr{L}^{-1} h\right)_{s}\right\|^{(0)}+\sum_{1}^{\infty}\left\|\left(\mathscr{T}^{n} \mathscr{L}^{-1} h\right)_{t}-\left(\mathscr{T}^{n} \mathscr{L}^{-1} h\right)_{s}\right\|^{(0)} \\
\leqq & c(T)\left[\frac{t-s}{\sqrt{s}}+\left\|b_{t}-b_{s}\right\|^{(1,0)}\right] \\
& +c(T)\left[\frac{(t-s)^{1-\varepsilon}}{\varepsilon}+\left\|b_{t}-b_{s}\right\|^{(1,0)}\right] \sum_{0}^{\infty}\left\|\mathscr{T}^{n} \mathscr{L}^{-1} h\right\|_{t}^{(2)}
\end{aligned}
$$

Moreover, if $\omega_{0} \in \mathscr{B}^{(2,1)}$ and $b \in \overline{\mathscr{B}}_{T}^{(2,0)}$, it is easy to show, proceeding as in the proof of Lemma 2.2 and Lemma 2.3, that

$$
\sum_{0}^{\infty}\left\|\mathscr{T}^{n} \mathscr{L}^{-1} h\right\|_{t}^{(2)} \leqq c(T)
$$

Estimates (D.40) and (D.41) immediately imply the lemma.

The last statement follows by (D.26).

\section{References}

1. Ladyzhenskaya, O.A.: The mathematical theory of viscous incompressible flows. New York: Gordon and Breach 1969

2. Temam, R.: Navier-Stokes equations. Amsterdam: North-Holland 1977

3. Ebin, D.G., Marsden, J.: Ann. Math. 92, 102 (1970) 
4. Kato, T.: J. Funct. Ann. 9, 296 (1972)

5. Marchioro, C.,Pulvirenti, M.:Vortex methods in two-dimensional fluid mechanics. In:Lecture Notes in Physics, No. 203. Berlin, Heidelberg, New York: Springer 1984

6. Batchelor, G.K.: An introduction to fluid mechanics. Cambridge: Cambridge University Press 1967

7. Chorin, A.J.: J. Fluid Mech. 57, 785 (1973)

8. Chorin, A.J., Hughes, T.J.R., McCracken, M.F., Marsden, J.E.: Commun. Pure Appl. Math. 31, 205 (1978)

9. Solonnikov, V.A.: Am. Math. Soc. Transl. 75, 1 (1968)

10. McCracken, M.F.: Siam J. Math. Ann. 12, 201 (1981)

Communicated by J. L. Lebowitz

Received December 27, 1983; in revised form June 15, 1984 
